\title{
On the Thermodynamics of Framework Breathing: A Free Energy Model for Gas Adsorption in MIL-53
}

\author{
An Ghysels, ${ }^{*}{ }^{\dagger} \ddagger$ Louis Vanduyfhuys, ${ }^{\dagger}$ Matthias Vandichel, ${ }^{\dagger}$ Michel Waroquier, $^{\dagger}$ \\ Veronique Van Speybroeck, ${ }^{\dagger}$ and Berend Smit ${ }^{\dagger}$ \\ ${ }^{\dagger}$ Center for Molecular Modeling, Ghent University, Technologiepark 903, 9052 Zwijnaarde, Belgium \\ ${ }^{\ddagger}$ Department of Chemical and Biomolecular Engineering, University of California, Berkeley, 101B Gilman Hall, Berkeley, California \\ 94720-1462, United States
}

\section{Supporting Information}

ABSTRACT: When adsorbing guest molecules, the porous metal-organic framework MIL-53(Cr) may vary its cell parameters drastically while retaining its crystallinity. A first approach to the thermodynamic analysis of this "framework breathing" consists of comparing the osmotic potential in two distinct shapes only (large-pore and narrow-pore). In this paper, we propose a generic parametrized free energy model including three contributions: host free energy, guest-guest interactions, and host-guest interaction. Free energy land-

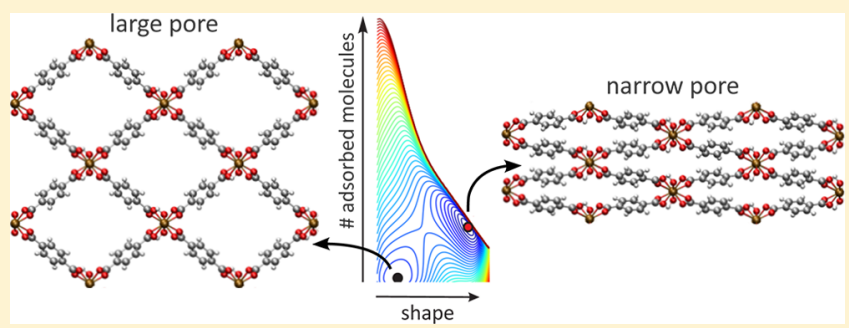
scapes may now be constructed scanning all shapes and any adsorbed amount of guest molecules. This allows us to determine which shapes are the most stable states for arbitrary combinations of experimental control parameters, such as the adsorbing gas chemical potential, the external pressure, and the temperature. The new model correctly reproduces the structural transitions along the $\mathrm{CO}_{2}$ and $\mathrm{CH}_{4}$ isotherms. Moreover, our model successfully explains the adsorption versus desorption hysteresis as a consequence of the creation, stabilization, destabilization, and disappearance of a second free energy minimum under the assumptions of a first-order phase transition and collective behavior. Our general thermodynamic description allows us to decouple the gas chemical potential $\mu$ and mechanical pressure $P$ as two independent thermodynamic variables and predict the complete $(\mu, P)$ phase diagram for $\mathrm{CO}_{2}$ adsorption in MIL-53(Cr). The free energy model proposed here is an important step toward a general thermodynamics description of flexible metal-organic frameworks.

\section{INTRODUCTION}

Metal-organic frameworks (MOFs) form an intriguing class of materials, whose extraordinary physicochemical and mechanical properties have attracted the attention of scientists over the last ten years. ${ }^{1,2}$ MOFs are porous crystalline materials with metal ions or clusters as the nodal points and organic linkers regularly arranged in between them. A large number of structures have been synthesized up to date due to the large variety in possible combinations of organic and inorganic subunits. ${ }^{3,4}$ Research has mainly focused on the discovery of new structures resulting in more than 10000 of these MOFs, illustrating the broad chemical versatility. ${ }^{5-7}$ Because of their large internal surface, the main promise of MOFs so far is in gas storage, purification, and separation. ${ }^{8-13}$

Some of these materials have remarkable stimuli-responsive behavior, i.e., their ability to undergo reversible, large-amplitude structural deformations under external physical stimuli such as temperature, ${ }^{14}$ mechanical pressure, ${ }^{15}$ light,${ }^{16}$ or gas or liquid exposure. ${ }^{4}$ The latter stimuli, the guest-induced structural transitions, have been widely studied in the literature and are often referred to as the "breathing" phenomenon. ${ }^{17}$

Only a limited number of MOFs show this extraordinary behavior (for example MIL- $88^{18}$ and MIL- $53^{19}$ ), where cell parameters may vary drastically, while the material retains its crystallinity. MIL-53 is the most commonly known framework exhibiting breathing. The structure is made up of cornersharing metal $\left(\mathrm{Cr},{ }^{19,20} \mathrm{Al}^{21} \mathrm{Fe}^{22,23} \mathrm{Ga}^{23}\right.$...) octahedral clusters interconnected with benzenedicarboxylate organic ligands, creating a three-dimensional structure with an array of onedimensional pore channels. The breathing was shown to be critically dependent on the metal at the nodal points ${ }^{24-26}$ as well as on the functionalization of the linkers. ${ }^{27,28}$ Figure 1 shows the two forms of MIL-53(Cr). At high temperature, MIL-53(Cr) takes the large pore (lp) form, but it may switch to its narrow pore $(\mathrm{np})$ variant with changing temperature or pressure or by adsorption of guest molecules such as $\mathrm{CO}_{2}{ }^{14,15}$ The two forms of the material have the same chemical composition and connectivity and differ only in their pore width. The structural changes have been observed with powder $X$-ray diffraction, ${ }^{29,30}$ neutron scattering, ${ }^{31}$ and infrared experiments, ${ }^{29}$ which showed a prominent hysteresis effect in the adsorption versus desorption isotherms. Computational simulations have been conducted with various guest species to

Received: November 25, 2012

Revised: April 22, 2013

Published: April 24, 2013 


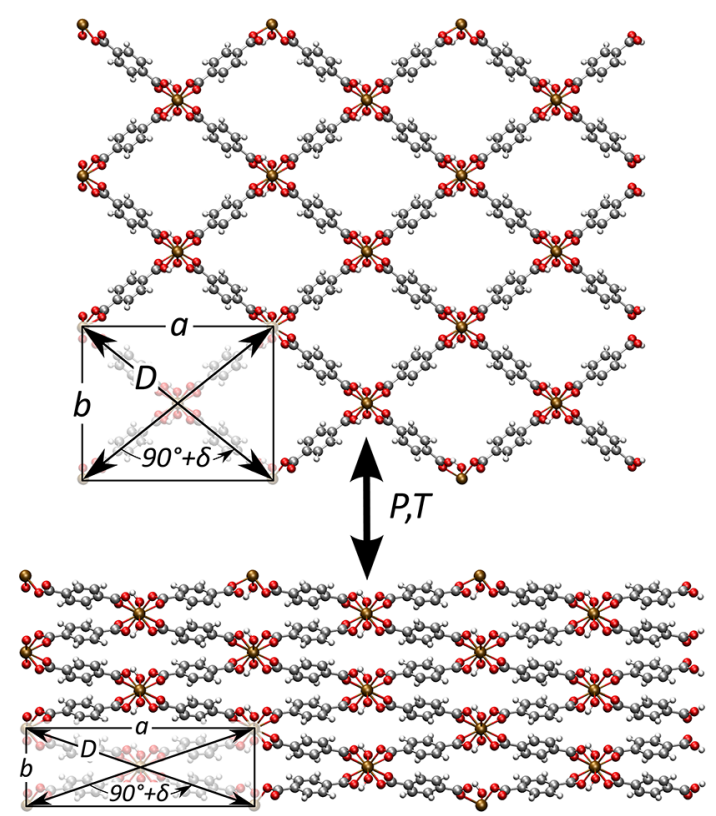

Figure 1. MIL-53 material with cell parameters $a, b, c$ has onedimensional channels (view along the $c$ axis). ${ }^{19}$ The material can switch between the large-pore (lp, upper figure) and narrow-pore (np, lower figure) shape under the influence of stimuli such as pressure $(P)$ and temperature $(T)$. The most flexible point was identified to be the connection between the inorganic chain and carboxylic function of the terephthalate. ${ }^{29}$ The diagonal $D$ in the $a, b$ plane corresponds to the length of two linkers and is approximately constant. The shape parameter $\delta$ is defined as the deviation from $\pi / 2$ of the angle between the diagonals. The color red represents oxygen, white hydrogen, gray carbon, and brown chromium.

investigate the bistable character using quantum and force-field calculations. $^{32-37}$

A great challenge consists in understanding the factors that govern these dynamic structural transitions. Theoretical models developed to study the thermodynamics of fluid adsorption usually consider the host matrix as a completely rigid framework, but structural transitions clearly require allowing shape changes in the model. Coudert et al. described the structural transition from a thermodynamic point of view, by comparing the grand potential in the osmotic ensemble for both the large pore and narrow pore forms based on experimental data. ${ }^{38}$ Neimark et al. continued this work and developed a stress-based model, by defining an osmotic stress which initiates the transitions. ${ }^{39}$ Triguero et al. proposed a deformation mechanism of the transition occurring via layer-bylayer shear, where subsequent two-dimensional layers are temporarily not in the same form. ${ }^{40}$ In other materials, the search for theoretical explanations of structural changes remains an active research question as well. For instance, Düren et al. investigated $\mathrm{N}_{2}$ adsorption isotherms in the zeolite ZIF- 8 with grand canonical Monte Carlo (GCMC) and explained the observed steps in the isotherms as a gating mechanism by linkers reorientation (no significant volume change), ${ }^{41}$ while Paesani et al. simulated the effect of the number of adsorbed benzene and isopropyl alcohol molecules on the volume and shape of the MOF material DMOF-1 with molecular dynamics. ${ }^{42}$ In those studies, a molecular explanation of the observed hysteresis is based on comparing the free energies of the two distinct $\mathrm{lp}$ and $\mathrm{np}$ forms, but intermediate shapes are not considered. Recently, a Wang-Landau-based Monte Carlo sampling method has been proposed to allow efficient sampling of the osmotic ensemble, which led to free energy landscapes as a function of the volume. ${ }^{43}$

In this paper, we propose a thermodynamic model to predict the conditions for structural transitions. In the osmotic ensemble, the thermodynamic control parameters are the amount of MOF material, the temperature, the gas chemical potential, and the mechanical pressure on the crystal. ${ }^{38} \mathrm{We}$ propose a generic free energy expression for this ensemble, which consists of a contribution from the host material, the guest molecules, and the host-guest interaction. This free energy shows the influence of the external chemical potential and external pressure, for a given host material and an adsorbate gas. Parametrization of an analytic expression for the free energy makes the simulation of free energy landscapes as a function of all shapes (not only the lp and np, but also intermediate shapes) computationally cheap. This allows us to determine in a straightforward fashion which phase of the material is most stable for any combination of external gas chemical potential and pressure and to rationalize which factors determine the experimentally observed hysteresis in the adsorption isotherm. The proposed thermodynamic model aims at (i) predicting whether a material breathes, (ii) reproducing the isotherm with the hysteresis on the structural transitions, and (iii) constructing the complete phase diagram in terms of the external chemical potential and pressure. The latter phase diagram has not been explored before because, in standard gas adsorption experiments, the chemical potential and pressure are not varied independently. From a theoretical point of view, the complete phase diagram gives insight into the factors contributing to breathing.

In section II, the osmotic ensemble is presented as the thermodynamic ensemble for adsorption-induced structural transitions. The external chemical potential and pressure are two independent variables for which a phase diagram with $\mathrm{lp}$ and $\mathrm{np}$ regions can be constructed. In section III, the shape descriptor is simplified from a strain tensor $\overline{\bar{\eta}}$ to a single parameter $\delta$. A free energy model is built up from three contributions: the empty host material, the interacting guest molecules, and the host-guest interactions. One of the key points in our rationale is the analytical function of the free energy profile of the empty host, based on the assumption of collective behavior for all unit cells of a crystal grain. The necessary model parameters are derived for MIL-53(Cr) with $\mathrm{CO}_{2}$ and $\mathrm{CH}_{4}$ as gas adsorbates, based on a combination of experimental data and simulated Monte Carlo data. These two gases are good examples to test the model because experiments indicate that $\mathrm{CO}_{2}$ causes breathing while $\mathrm{CH}_{4}$ does not at room temperature. ${ }^{30}$ Moreover, MIL-53(Cr) has been proposed for the separation of these two fuel combustion gases. ${ }^{44}$ In section IV, our free energy model is applied to predict the phase behavior in an isotherm measurement. The proposed mechanism of creation, stabilization, destabilization, and disappearance of a second minimum in the free energy surface explains the hysteresis effect between adsorption and desorption. Our reasoning is not based on a threshold thermal average energy that should be available to overcome the barrier (as proposed by Miyahara et al. $^{45}$ or Bousquet et al. ${ }^{43}$ ) but assumes a first-order phase transition characterized by a collective transition of the system when the barrier disappears. Carbon dioxide is correctly predicted to make MIL-53(Cr) breathe, while methane is not, in accordance with experiment. This demonstrates that our model encompasses the key factors 
that cause breathing. Next the complete $(\mu, P)$ phase diagram is constructed. Finally the influence of the model parameters on this phase diagram is investigated. We expect that our methodology is generally applicable and able to predict whether a material breathes and at what conditions of gasphase chemical potential and external pressure.

\section{THERMODYNAMICS: THE OSMOTIC ENSEMBLE}

A. Constant $(n, \mu, P, T)$ Ensemble. The thermodynamics of gas adsorption in a flexible porous material are governed by the following thermodynamical variables: the amount of host material, which we express by the number of unit cells of the host material $(n)$, the chemical potential of the adsorbed gas molecules $(\mu)$, the mechanical stress tensor (Cauchy stress tensor $\overline{\bar{\sigma}}$ ), and the temperature $(T)$ (see Figure 2). It is

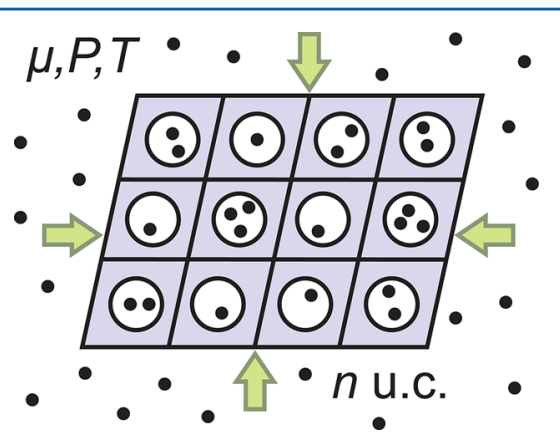

Figure 2. Adsorption of gas molecules in the pores and channels of the framework is described by the osmotic ensemble $(n, \mu, P, T)$ with constant number $n$ of unit cells (u.c.) of the porous host material, constant chemical potential $\mu$ of the guest molecules, constant pressure $P$ (gas pressure and/or mechanical pressure), and constant temperature $T$. The relation between the stress tensor $\overline{\bar{\sigma}}$ and pressure $P$ is given by $\sigma_{i j}=-P \delta_{i j}$ in the standard adsorption experiments.

instructive to compare this general setup with the typical experimental setup for measuring an isotherm, in which, for example, the weight of the sample is measured as a function of the pressure. In such a system, the stress is equal to (the negative of) the isotropic gas, and the gas pressure is coupled to the chemical potential through the equation of state. However, in addition to this gas pressure one can apply an additional force. For example, in geological formation such stresses are very common, and these forces are completely independent of gas pressure. Poromechanics is another example where chemical potential and pressure are typically decoupled. ${ }^{46}$ In this paper, the stress is caused by an isotropic gas pressure $P\left(\sigma_{i j}\right.$ $=-P \delta_{i j}$, see Supporting Information (S.I.)), unless stated otherwise. The resulting constant $(n, \mu, P, T)$ ensemble is an example of an osmotic ensemble (Figure 2). ${ }^{47-49}$

The term osmotic ensemble is generally used for a twocomponent system where one component has a constant amount of molecules, while the other has a constant chemical potential. In the case of gas adsorption in MOFs, the amount of MOF material and the chemical potential of the gas are kept constant. The osmotic thermodynamic potential, here denoted by $X(n, \mu, P, T)$, is the free energy of the ensemble and can be seen as a generalization of the grand canonical potential, where besides particle insertions also volume changes caused by the structural transitions are allowed. In section III an analytical model is proposed for this free energy.

B. Gas Adsorption Experiments. Since the discovery of MIL-53(Cr) in 2002, ${ }^{19}$ its phase behavior has been investigated thoroughly using adsorption experiments. In vacuum, the observed MIL-53(Cr) structure at $300 \mathrm{~K}$ is the large-pore (lp) shape. In an adsorption experiment, the material is brought in a chamber filled with gas. The gas pressure is gradually increased while keeping the temperature constant. The amount of gas molecules adsorbed in the pores of MIL-53 is monitored as a function of the pressure using gravimetrical or volumetrical measurements.

For instance, when bringing the material in a chamber filled with $\mathrm{CO}_{2}$ gas at approximately 1 bar and at $304 \mathrm{~K}$, the structure adsorbs gas molecules and shrinks to its narrow-pore (np) shape. It corresponds with a first plateau in the isotherm. However, the structure returns to its original $\mathrm{lp}$ shape when the $\mathrm{CO}_{2}$ pressure is brought above approximately 6 bar. On the isotherms this is manifested by a sudden increase of the adsorption ending with a second plateau. In the desorption process a slightly different behavior is noticed due to hysteresis. Both $\mathrm{lp} \rightarrow \mathrm{np}$ and $\mathrm{np} \rightarrow \mathrm{lp}$ transitions occur at lower pressures in the desorption process.

The situation is different for an adsorption experiment with $\mathrm{CH}_{4}$ gas, where no such breathing is observed at room temperature. ${ }^{30}$ The structure retains its $1 p$ phase. The difference between $\mathrm{CO}_{2}$ and $\mathrm{CH}_{4}$ adsorption lies in the interactions of the gas molecules with the host material. According to microcalorimetric experiments, the heat of adsorption is comparable for $\mathrm{CO}_{2}$ and $\mathrm{CH}_{4}$ in the lp phase, but the adsorption of $\mathrm{CO}_{2}$ is much more favorable than the adsorption of $\mathrm{CH}_{4}$ in the $\mathrm{np}$ phase. $^{50}$

C. $(\mu, P)$ Phase Diagram. In the standard isotherm adsorption experiments, the chemical potential of the gas $(\mu)$ is directly related to the gas pressure $(P)$ in the chamber through the equation of state of the gas: $P=P(\mu, T)$. Nonetheless, in variations of the experiment, the chemical potential and pressure on the MOF material can be decoupled such that they become independent control variables. For instance, an additional mechanical pressure can be realized by pressing the MOF crystal between diamond tips. Alternatively, shear stress can be generated by attaching the crystal between two substrates (or embedding in mesoporous matrices) and pulling the substrates in opposite directions, possibly giving an anisotropic Cauchy stress tensor.

In our thermodynamic analysis, the chemical potential and pressure are considered as two independent variables as well. Determining the "phase" (lp or $\mathrm{np}$ ) for each combination of $\mu$ and $P$ leads to the complete $(\mu, P)$ phase diagram at constant $T$. The equation of state is then a single line on the phase diagram. The decoupling of $\mu$ and $P$ is helpful in extending the osmotic ensemble to chemical potentials of mixtures ${ }^{44,51}$ and partial pressures in a thermodynamically correct way.

We propose the hypothetical scheme in Figure 3 for the phase diagram of a breathing material. The large-pore shape is assumed to be the equilibrium structure in the absence of molecules ( $\mu$ very low) and pressure $(P=0)$, which corresponds to the left bottom corner of the phase diagram. Let us discuss three cuts through the surface.

In the horizontal cut (1), the chemical potential is kept constant at a very low value, such that the host is basically empty. Experimentally, one would apply a mechanical stress on the host material in vacuum. By applying external stress (i.e., squeezing the material), the structure eventually collapses at a certain transition pressure $P_{\mathrm{t}}$ to attain its narrow-pore shape. However, along a horizontal cut $\left(1^{\prime}\right)$ at higher chemical potential, where adsorbate molecules fill up the pores, the 


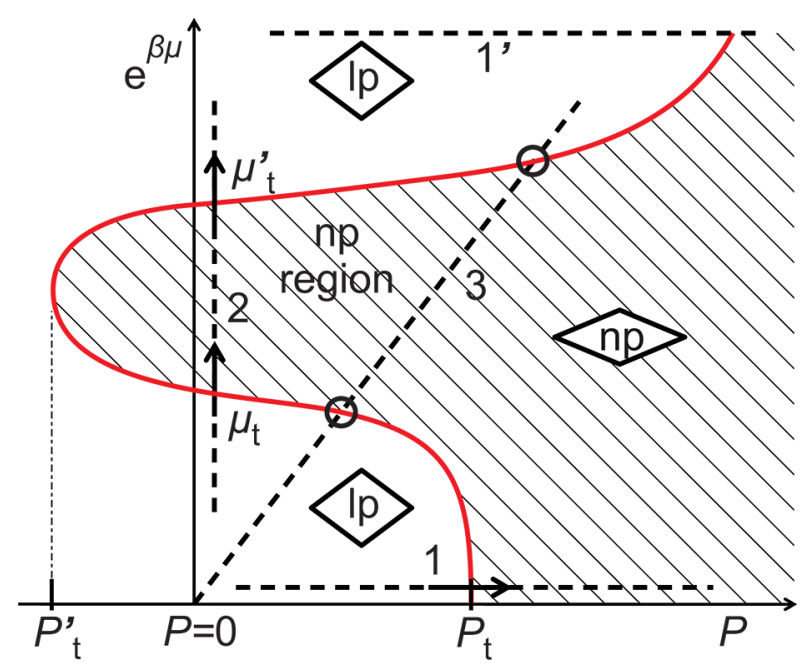

Figure 3. Hypothetical $(\mu, P)$ phase diagram of breathing framework at constant $T$ and constant number of unit cells, with $\exp (\beta \mu)$ on the vertical axis, $\beta=1 / k_{\mathrm{B}} T$. Solid red line indicates the border between the large-pore (lp) and narrow-pore (np) phase. Dashed lines are three cuts: (1) constant $\mu$ ( 1 is empty, $1^{\prime}$ is full), (2) constant $P$, (3) equation of state $\mu(P)$ of an ideal gas. Transition pressures $P_{\mathrm{t}}$ and $P_{\mathrm{t}}^{\prime}$ and transition chemical potentials $\mu_{\mathrm{t}}$ and $\mu_{\mathrm{t}}^{\prime}$ lie on the border between the $\mathrm{lp}$ and $\mathrm{np}$ regions.

pressure can reach high values while remaining in the lp phase. This explains why the lp phase extends quite far to the right in the upper right corner of the phase diagram.

In the vertical cut (2), the pressure is kept constant, while the chemical potential varies. Experimentally, one could achieve this by varying the gas pressure such that the chemical potential varies, while an extra mechanical pressure is executed on the material to keep the total pressure constant. If the adsorption of molecules in the narrow-pore structure is more favorable than in the large-pore structure, the structure will collapse at some transition chemical potential $\mu_{\mathrm{t}}$. The higher the chemical potential, the higher the number of adsorbed molecules. Once the narrow is fully filled and the repulsion between the adsorbed molecules is too high, it becomes more favorable for the molecules to adsorb in the lp phase than in the np phase. In such a scenario, the large-pore shape is favored to accommodate even more molecules, such that a second transition occurs at $\mu_{\mathrm{t}}^{\prime}$. The intermediate np region depends clearly on the balance between the favorable interactions and the available pore volume. If the interactions between host and guest molecules are too weak, the first transition at $\mu_{\mathrm{t}}$ does not take place, and the $\mathrm{np}$ region is absent. If the interactions are too strong, the first transition at $\mu_{\mathrm{t}}$ occurs at extremely low chemical potential, and the np region extends to the bottom of the phase diagram. If the temperature increases, the $n p$ region is expected to become smaller: the entropic effects, which scale with temperature and in general favor bigger volumes, i.e., the lp phase, can become stronger than the energetic effects of the guest-host interactions. The presence of the $\mathrm{np}$ region surrounded by a $\mathrm{lp}$ region is a condition for the occurrence of the breathing effect.

The last cut (3) mimics the standard experimental situation in which the external pressure is equal to the gas pressure. In this case the pressure and the chemical potential cannot be changed independently but are related through the equation of state $\mu(P, T)$ of the gas in the gas chamber. The cut on the plot represents the equation of state of an ideal gas, for which $\mu=\mu_{0}$
$+k_{\mathrm{B}} T \ln P / P_{0}$, with $\left(\mu_{0}, P_{0}\right)$ being the values at a reference state, $k_{\mathrm{B}}$ the Boltzmann constant, and $T$ the temperature. With increasing pressure (or, equivalently, increasing chemical potential), two transitions occur: $\mathrm{lp} \rightarrow \mathrm{np}$ followed by $\mathrm{np} \rightarrow \mathrm{lp}$. These two transitions are usually referred to as the breathing effect. These results show that for such a system the condition for the breathing effect is the equation of state that intersects with the np region.

One could also imagine negative pressures, which would correspond to pulling the framework crystal isotropically. No such experimental setup for MIL-53 has been designed so far, but it is important to note that these negative pressures are experimentally accessible. One could envision that these materials are embedded in a polymer film. In such membranes such negative pressures can occur. It seems reasonable that the $\mathrm{np}$ region disappears on the left-hand side beyond a certain threshold negative pressure $P_{\mathrm{t}}^{\prime}$. Indeed, when "pulling" the crystal strongly enough, the material will open even when the molecules inside the pores prefer to adsorb in the narrow-pore shape.

D. Collective Phase Transitions. Intrusion experiments performed with MIL-53(Cr) give us additional viable information about the thermodynamics of the empty crystals. The crystal powder is brought under isotropic stress (pressure $P)$ by dispersing the powder in mercury liquid and subsequently pressing the liquid. As mercury molecules are too large to enter the pores, the measured volume change must be caused by compression of the empty MIL-53 unit cells. Beurroies et al. and later Neimark et al. found an $\mathrm{lp} \rightarrow \mathrm{np}$ transition at $(550 \pm 150)$ bar and the reverse $n p \rightarrow l p$ transition at $(100 \pm 100)$ bar. $^{15,52}$ The discontinuous shape transition is accompanied by a hysteresis loop, suggesting that the crystal undergoes a first-order phase transition (cf. Landau theory in magnetism) and collective behavior. We now briefly discuss the impact of these two suggested properties.

The first-order phase transition is induced by the pressure as a control variable. Under the influence of increasing pressure, the $P V$ term in the free energy can destabilize the lp shape. The discontinuous character of the transition implies that the free energy profile $F_{\text {host }}$ of the empty host as a function of volume has two inflection points $\left(\partial^{2} F_{\text {host }} / \partial V^{2}=0\right)$ located between the $\mathrm{lp}$ and $\mathrm{np}$ volumes. The region between these inflection points is then unstable $\left(\partial^{2} F_{\text {host }} / \partial V^{2}<0\right)$.

A single-crystal grain in the MIL-53 powder can be regarded as a system consisting of unit cells that have to transition simultaneously because of geometrical constraints. It is not possible for this periodic structure that a few unit cells take the lp shape, while neighboring unit cells take the np shape, except in a layered organization. The existence of an intermediate lp/ $\mathrm{np}$ shape where the $\mathrm{lp}$ and $\mathrm{np}$ shapes alternate in layers has indeed been suggested by, e.g., force field calculations by Ghoufi et al. ${ }^{53}$ Because of the geometrical constraints, the transition between the lp shape, np shape, and intermediate layered shape should take place collectively for all unit cells within each layer. Triguero et al. wrote a clear, illustrated discussion how phase transformations of the MIL-53 topology should occur in a cooperative manner and necessarily involve an entire layer of cells, all of which must be in the same shape. ${ }^{40}$ The present paper adopts a single unit cell as a building block of the periodic structure and thus does not support the situation with alternating $\mathrm{lp} / \mathrm{np}$ layers. Due to this simplification, these intermediate shapes are neglected, and the assumption of collective behavior results in an identical shape 
for all unit cells. Nevertheless, our model could be extended toward the description of super cells and mixed $\mathrm{lp} / \mathrm{np}$ phases (i.e., by the introduction of additional shape parameters in section III).

Collective behavior can lead to hysteresis as follows. Assume MIL-53 is in the lp shape at zero pressure, meaning that the lp shape is a local minimum of the free energy profile. The $P V$ term may create or stabilize a second minimum at the np phase: with increasing pressure, it becomes more favorable for the system to take a smaller volume. When the np phase becomes more stable than the lp phase, the $\mathrm{lp} \rightarrow \mathrm{np}$ transition can not immediately take place, because collective behavior of the periodic system is assumed: all unit cells have to transition simultaneously. Even the tiniest free energy barrier per unit cell represents a huge barrier for the total crystal grain. The transition is then only possible when the free energy barrier disappears, i.e., as soon as the increasing pressure makes the lp phase no longer be a local free energy minimum, pushing the system into the np phase. Similarly, decreasing the pressure will cause the reverse $n p \rightarrow l p$ transition when the $n p$ phase is no longer a free energy minimum state. The hysteresis then follows from the difference in pressure when the lp phase versus the $n p$ phase disappear.

Outgassed (empty) MIL-53(Cr) crystals take the lp shape at approximately $300 \mathrm{~K} \cdot{ }^{29,30,37,50}$ This implies that there is only one minimum (lp shape) in the free energy profile as a function of volume. Indeed, if a second minimum (np shape) were to be present, hysteresis could cause the system to remain in the $n p$ shape at $300 \mathrm{~K}$. As only the lp shape is found experimentally, the profile should have a sole minimum at the lp phase. Note that a one-well potential at $300 \mathrm{~K}$ is not in contradiction with the two-well potentials that have been calculated in the literature quantum mechanically at $0 \mathrm{~K}$. The $0 \mathrm{~K}$ calculations are missing the entropy contribution, which might stabilize the lp shape with increasing temperature and make the $n p$ well disappear at some transition temperature.

\section{FREE ENERGY MODEL}

The aim is to construct a free energy model that shows the influence of the external pressure and the external chemical potential, given a specific host material and an adsorbate gas. As it is the intention to include all intermediate shapes in the description (not only the lp and $\mathrm{np}$ shapes), an analytical function is proposed for the osmotic potential that depends on the shape of the unit cell and the number of adsorbed molecules. First, the shape description is simplified from a tensor quantity (the strain tensor) to a single scalar parameter $(\delta)$. Next, the free energy $F$ is assumed to consist of a contribution from the host material, the adsorbed molecules, and the interaction between host material and adsorbed molecules. The three contributions to the free energy are discussed as a function of this parameter $\delta$. The functional form of the free energy is then parametrized by fitting to experimental and simulated data. The key parameters are the flexibility of the empty host material, the equation of state of the gas, and the interaction of the adsorbate molecules with the host. Finally, a Legendre transform makes the transition to the osmotic $(n, \mu, P, T)$ ensemble.

A. Shape Description. In contrast to liquids, solids resist shear. Their stress and strain state are not guaranteed to be isotropic but need to be expressed by a tensor quantity. In continuum mechanics, the deformation of a body near a reference point is characterized by the Lagrangian finite strain tensor $\overline{\bar{\eta}}$ (also called Green's strain tensor)

$$
\overline{\bar{\eta}}=\frac{1}{2}\left(\overline{\bar{\alpha}}^{T} \overline{\bar{\alpha}}-1\right)
$$

where $\overline{\bar{\alpha}}$ is the deformation gradient tensor, with as components the derivatives of the deformed material positions $x$ with respect to the reference material position $X$

$$
\alpha_{i j}=\frac{\partial x_{i}}{\partial X_{j}}
$$

where $i, j$ are Cartesian indices. The tensor $\overline{\bar{\eta}}$ has dimension $3 \times$ 3 and is symmetric ( $\overline{\bar{\alpha}}$ is not), such that the shape can be described by six elements of $\overline{\bar{\eta}}$.

The benzene-dicarboxylate linkers are quite rigid units. Indeed, the diagonal $D$ in the $(a, b)$-plane of the unit cell (Figure 1) corresponds to two linker lengths and takes experimental values of 21.213 and $21.192 \AA$ for the lp and $n p$ phase of MIL-53(Cr), respectively. ${ }^{19}$ It is thus justified to assume a constant length for the diagonal. ${ }^{43}$ The cell vector in the $c$-direction remains approximately constant as well. ${ }^{19}$ Moreover, we assume that the unit cell stays orthorhombic while deforming. According to experiment, the $\beta$ angle changes by $15^{\circ},{ }^{19}$ making the np unit cell monoclinic, but this change is neglected in our model by keeping the angles $\alpha, \beta$, and $\gamma$ constant. Under these assumptions, only one degree of freedom can change in the orthorhombic unit cell: cell vectors $a$ and $b$ will adapt their relative length to obey the constraint of the constant diagonal as $a^{2}+b^{2}=D^{2}, D$ constant. Hence, in the present model only one degree of freedom out of six is kept to treat the flexibility of the framework, which facilitates the further model development.

We introduce the shape parameter $\delta$, which is defined as the deviation from $90^{\circ}$ between two diagonals in the $(a, b)$ plane of the unit cell (Figure 1). $\delta$ lies in the range $-\pi / 2$ to $\pi / 2$ : the two extreme values correspond to a fully collapsed framework. Because the free energy diverges in the fully collapsed framework, we construct a polynomial model for $F$ in $\tan \delta$. The limiting behavior $F \rightarrow \infty$ when $\delta \rightarrow \pm \pi / 2$ is then easily imposed by making the coefficient of the highest power (even) in $\tan \delta$ strictly positive.

For MIL-53, three shapes play a role in this story (details in S.I.): the reference shape where $a=b=a_{0}(\delta=0)$, the largepore shape with $a / b=1.3\left(\delta=14^{\circ}, \tan \delta=0.25\right)$, and the narrow-pore shape with $a / b=2.7\left(\delta=46^{\circ}, \tan \delta=1.05\right)$. The first has tetragonal symmetry and is taken as the reference state with volume $V_{0}$, while the last two shapes are orthorhombic and are the experimentally observed ones. The volume $V$ of a particular shape is related to the shape parameter $\delta$ by the equation $V=V_{0} \cos \delta$. Note that the $\mathrm{OH}$ groups attached to the metal sites and the relative position of the linkers break the symmetry, so shapes $\delta$ and $-\delta$ represent different structures despite having the same volume. The reference shape $\delta=0$ has thus no special physical meaning (no symmetry point).

B. Free Energy. The system under consideration consists of $n$ unit cells of porous host material wherein $N$ guest molecules are adsorbed at a temperature $T$. We assume that the free energy of a porous material in shape $\delta$ with $N$ adsorbed molecules can be expressed as the sum of three contributions: the empty host material $\left(F_{\text {host }}\right)$, the $N$ interacting guest molecules in the pore volume $\left(F_{N}\right)$, and the interaction between the host and guest molecules $\left(F_{\text {int }}\right)$ 


$$
\begin{aligned}
F(n, N, \delta, T)= & F_{\text {host }}(n, \delta, T)+F_{N}(n, T, \delta, T) \\
& +F_{\text {int }}(N, \delta, T)
\end{aligned}
$$

In reality the Hamiltonian is a function of the positions of both the host atoms and the guest atoms. Imposing that the energy of the host is independent of the precise position of the guest atoms, and vice versa, that the guest energies are independent of the host atom positions, implies the separation in three parts in eq 3.

C. Free Energy $F_{\text {host }}$ of the Empty Host. $F_{\text {host }}$ is the free energy contribution of the empty host material as a function of its shape. We postulate a profile consistent with the experimental observations: lp is most stable at $300 \mathrm{~K}$, and hysteresis occurs for structural changes induced by mechanical pressure. As explained in section IID, these observations suggest a free energy profile as a function of volume with one minimum at the lp shape and two inflection points, when the pressure-induced hysteresis is regarded as a first-order and collective phase transition. The following fourth-order polynomial in $\tan \delta$ is proposed

$$
\begin{aligned}
F_{\text {host }}(n, \delta, T)= & n\left(k_{1} \tan \delta+k_{2} \tan ^{2} \delta+k_{3} \tan ^{3} \delta\right. \\
& \left.+k_{4} \tan ^{4} \delta\right)
\end{aligned}
$$

where the free energy of the empty structure in the reference shape $\delta=0$ serves as the reference. The coefficients $k_{i}$ are temperature dependent. The parameters express the resistance to changes in shape (the flexibility of the material). The parameter $k_{4}$ should be strictly positive to ensure the correct divergence $\left(F_{\text {host }} \rightarrow \infty\right)$ when the structure fully collapses $(\delta \rightarrow$ $\pm \pi / 2)$.

The four parameters are fitted to allow for the following scenario for the empty host material. The lp phase is stable at zero pressure $\left(P=-\partial F_{\text {host }} / \partial V=0\right.$ at $\left.V_{\text {lp }}\right)$, while a transition to the $n p$ phase is observed at higher pressure, with some hysteresis on this transition when reverting the process. This has been experimentally observed by Beurroies et al. with mercury intrusion measurements on MIL-53(Cr). ${ }^{15,52}$ Due to the high wetting angle, mercury does not enter the pores, but nevertheless the fluid exerts an external mechanical pressure on the crystals. The transition $l p \rightarrow n p$ and reverse transition $n p \rightarrow$ lp were measured to take place at approximately $P_{1}=(550 \pm$ 150) bar and $P_{2}=(100 \pm 100)$ bar, respectively. ${ }^{52}$ The corresponding transition volumes were measured to be approximately $V_{1}=1200 \AA^{3}$ and $V_{2}=1400 \AA^{3}$ (S.I. of ref.52). The $F_{\text {host }}$ profile may reproduce this hysteresis if two inflection points $\left(\partial^{2} F_{\text {host }} / \partial V^{2}=0\right)$ are imposed at these volumes and pressures. Moreover, calorimetric measurements give a free energy difference of about $5 \mathrm{~kJ} / \mathrm{mol}$ between the $\mathrm{lp}$ and np phase. ${ }^{38}$ Coombes et al. calculated the energy quantum mechanically at the experimental $\mathrm{lp}$ and $\mathrm{np}$ cell parameters and found an energy difference of $42 \mathrm{~kJ} / \mathrm{mol}$ at $0 \mathrm{~K}^{34}$ Civalleri et al. found a quantum mechanical energy difference in the range of $39-42 \mathrm{~kJ} / \mathrm{mol}$ at $0 \mathrm{~K}$ for the fully relaxed structures of the MIL$53(\mathrm{Al})$ using a variety of Grimme corrected density functionals and estimated the entropy controbution from phonon calculations to be around $16 \mathrm{~kJ} / \mathrm{mol}$ at $300 \mathrm{~K}$, giving a free energy difference of approximately $23-26 \mathrm{~kJ} / \mathrm{mol}$ for MIL$53(\mathrm{Al}) .{ }^{35}$ Bousquet et al. postulated a difference of $7.5 \mathrm{~kJ} / \mathrm{mol}$ and a barrier of $15 \mathrm{~kJ} / \mathrm{mol}$ at $300 \mathrm{~K}^{43}$ In this paper, we use a free energy difference between volumes $V_{1}$ and $V_{2}$ of at least $\Delta F_{12}=10 \mathrm{~kJ} / \mathrm{mol}$, which is acceptable given the previous findings in the literature and which gave transition pressures close to the $P_{1}$ and $P_{2}$ values in the fitting procedure (vide infra). We are aware there is a given degree of arbitrariness in the choice of $\Delta F_{12}$, and further investigation is recommended to study the impact of the host profile on the final adsorption induced hysteresis properties.

Six constraints are now imposed: (1) the lp structure is stable at zero pressure; $(2-3)$ the $F_{\text {host }}$ has two inflection points at the transition volumes $V_{1}$ and $V_{2} ;(4-5)$ the pressure at these inflection points is $P_{1}$ and $P_{2}$; and (6) the free energy barrier between the lp and np phase is approximately $\Delta F_{12}$. Fitting the four parameters in eq 4 with these six constraints leads to the

\begin{tabular}{|c|c|c|c|c|}
\hline term & parameters & & & fitted function \\
\hline$F_{\text {host }}$ & $k_{1} \mathrm{~kJ} / \mathrm{mol}$ & & fou & th-order polynomial in $\tan \delta$ \\
\hline & $k_{2} \mathrm{~kJ} / \mathrm{mol}$ & 651.81 & & \\
\hline & $k_{3} \mathrm{~kJ} / \mathrm{mol}$ & -787.20 & & \\
\hline & $k_{4} \mathrm{~kJ} / \mathrm{mol}$ & 327.46 & & \\
\hline term & parameters & $\mathrm{CO}_{2}$ & $\mathrm{CH}_{4}$ & fitted function \\
\hline$V_{\mathrm{p}}$ & $f_{0}, \AA^{3}$ & 803.66 & 779.14 & $\begin{array}{l}\text { sixth-order polynomial in } \\
\quad \tan \delta\end{array}$ \\
\hline & $f_{1}, \AA^{3}$ & -125.68 & -123.95 & \\
\hline & $f_{2}, \AA^{3}$ & -526.67 & -466.82 & \\
\hline & $f_{3}, \AA^{3}$ & 62.17 & 59.14 & \\
\hline & $f_{4}, \AA^{3}$ & 114.90 & 81.50 & \\
\hline & $f_{5}, \AA^{3}$ & -7.81 & -7.02 & \\
\hline & $f_{6}, \AA^{3}$ & -8.27 & -3.63 & \\
\hline$F_{N}$ & a, $\AA^{3} \mathrm{~kJ} / \mathrm{mol}$ & 611.87 & 386.62 & van der Waals coefficients \\
\hline & $b, \AA^{3}$ & 71.24 & 71.57 & \\
\hline$\Delta U$ & $c_{0}, \mathrm{~kJ} / \mathrm{mol}$ & -20.27 & -18.20 & $\begin{array}{l}\text { sixth-order polynomial in } \\
\quad \tan \delta\end{array}$ \\
\hline & $c_{1}, \mathrm{~kJ} / \mathrm{mol}$ & 3.10 & 1.48 & \\
\hline & $c_{2}, \mathrm{~kJ} / \mathrm{mol}$ & -10.93 & -5.54 & \\
\hline & $c_{3}, \mathrm{~kJ} / \mathrm{mol}$ & -3.47 & -5.26 & \\
\hline & $c_{4}, \mathrm{~kJ} / \mathrm{mol}$ & -9.29 & -11.24 & \\
\hline & $c_{5}, \mathrm{~kJ} / \mathrm{mol}$ & 2.77 & 3.59 & \\
\hline & $c_{6}, \mathrm{~kJ} / \mathrm{mol}$ & 3.48 & 6.06 & \\
\hline
\end{tabular}
values in Table 1 , and the fitted $F_{\text {host }}$ is displayed in Figure 4.

Table 1. Fitted Coefficients in the Free Energy Model for $\mathrm{CO}_{2}$ and $\mathrm{CH}_{4}$ Adsorption in MIL-53(Cr) at $300 \mathrm{~K}^{a}$

${ }^{a}$ Parameters in the host free energy $F_{\text {host }}$ (eq 4$)$ are independent of the choice of guest molecules. The pore volume $V_{\mathrm{p}}$ (eq 7), interaction $\Delta U$ (eq 8$)$, and van der Waals parameters $(a, b)$ are different for $\mathrm{CO}_{2}$ and $\mathrm{CH}_{4}$.

Not all constraints may be satisfied simultaneously, and some deviations are noticed. For instance, the transition pressures in the fitted profile are 200 and 1100 bar. The free energy difference $\Delta F_{12}$ is $10.7 \mathrm{~kJ} / \mathrm{mol}$, while the difference between the experimental lp and $\mathrm{np}$ shape is $21.3 \mathrm{~kJ} / \mathrm{mol}$. The S.I. gives in addition the pressure profile and the second derivative profile as a function of volume, illustrating the instability region $\left(\partial^{2} F_{\text {host }} /\right.$ $\left.\partial V^{2}<0\right)$ between the two inflection points. We noticed that the resulting profile is sensitive to the specific value of the parameters $V_{1}, V_{2}, P_{1}, P_{2}$, and $\Delta F_{12}$, which is thus a weak point in the model development. In the future, significant improvement could be made if quantum mechanical free energy profiles were available that provide a reliable entropy estimation including anharmonicity effects as a function of shape.

The quality of our host free energy profile may be evaluated by comparing the bulk modulus, defined as $K=-V \partial P / \partial V$, with experimental data. Serra-Crespo et al. report a bulk modulus of $10.9 \mathrm{GPa}$ for the $\mathrm{NH}_{2}-$ MIL-53(In) material. ${ }^{54}$ Neimark et al. 


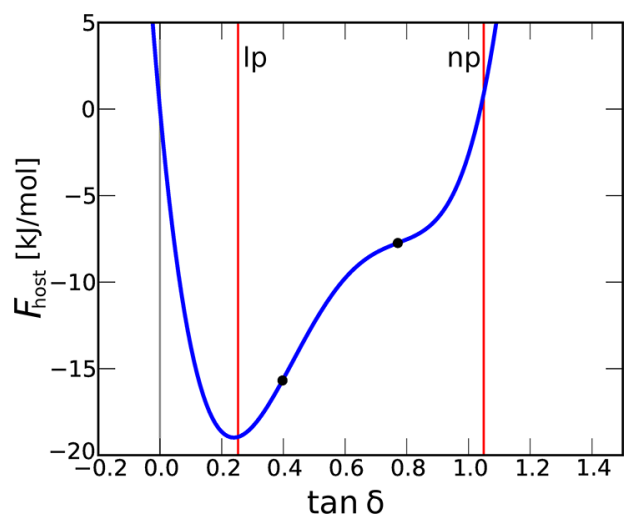

Figure 4. Host free energy profile $F_{\text {host }}[\mathrm{kJ} / \mathrm{mol}]$ of one empty MIL$53(\mathrm{Cr})$ unit cell as a function of the shape at $300 \mathrm{~K}$. The proposed curve is a fourth-order polynomial in $\tan \delta$ (eq 4) with two inflection points (black dots). The vertical lines (red) indicate the shape of the experimentally observed $\mathrm{lp}$ and $\mathrm{np}$ structures.

find a bulk modulus equal to $2 \mathrm{GPa}$ for the lp shape and 10 GPa for the np shape of MIL-53(Cr) at a temperature of 300 $\mathrm{K}^{52}$ Ortiz et al. calculated bulk moduli in the range 1-20 GPa for a range of flexible porous materials at $0 \mathrm{~K} .{ }^{55}$ Our profile $F_{\text {host }}$ at $300 \mathrm{~K}$ gives a bulk modulus of $8.7 \mathrm{GPa}$ in the lp shape, which has an acceptable order of magnitude.

D. Free Energy $F_{N}$ of $N$ Molecules. $F_{N}$ is the free energy of $N$ gas molecules enclosed in the total pore volume $n V_{\mathrm{p}}$, where $n$ is the number of unit cells and $V_{\mathrm{p}}=V_{\mathrm{p}}(\delta)$ is the pore volume of a single unit cell, which depends on the shape $\delta$ of the unit cell. When the gas is well described by the equation of state of a van der Waals gas, the term can be written as the wellknown free energy expression

$$
\begin{aligned}
F_{N}(n, N, \delta, T)= & k_{\mathrm{B}} T \ln N ! \\
& -N k_{\mathrm{B}} T \ln \left(\frac{P_{0}\left(n V_{\mathrm{p}}(\delta)-b N\right)}{k_{\mathrm{B}} T}\right) \\
& -a \frac{N^{2}}{n V_{\mathrm{p}}(\delta)}+N \mu_{0}(T)
\end{aligned}
$$

In eq $5, a$ and $b$ are the van der Waals parameters; $P_{0}$ is a reference pressure (e.g., 1 bar); and $\mu_{0}(T)$ is a reference potential, here the chemical potential of an ideal gas at temperature $T$ and pressure $P_{0}$. The logarithmic of $n V_{\mathrm{p}}-b N$ ensures that the maximum number of molecules in a single unit cell is below $V_{\mathrm{p}} / b$. This corresponds with the idea that the van der Waals parameter $b$ represents the volume taken by one adsorbate molecule.

The pore volume $V_{\mathrm{p}}$ has been derived from Monte Carlo simulations with Widom insertions for a range of shapes. ${ }^{56}$ The pore volume fraction $V_{\mathrm{p}} / V$ is derived from the Rosenbluth factor using $\mathrm{CO}_{2}$ or $\mathrm{CH}_{4}$ as a probe (details in S.I.). Next a sixth-order polynomial in $\tan \delta$ has been fitted, which serves as input in the free energy model

$$
\begin{aligned}
V_{\mathrm{p}} & =f_{0}+f_{1} \tan \delta+f_{2} \tan ^{2} \delta+f_{3} \tan ^{3} \delta+f_{4} \tan ^{4} \delta \\
& +f_{5} \tan ^{5} \delta+f_{6} \tan ^{6} \delta
\end{aligned}
$$

The parameters are given in Table 1 and Figure 5 show the Monte Carlo pore volume $V_{\mathrm{p}}$ with the fitted polynomial.

E. Interaction Energy $F_{\text {int }} \cdot F_{\text {int }}$ is the interaction of the $N$ absorbed molecules with the host material. Each adsorbate

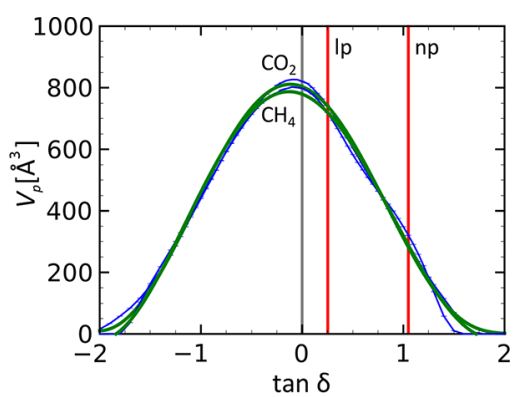

Figure 5. Pore volume $V_{\mathrm{p}}\left[\AA^{3}\right]$ available for $\mathrm{CO}_{2}$ and $\mathrm{CH}_{4}$ in MIL$53(\mathrm{Cr})$ as a function of the shape. Monte Carlo data (blue, with Monte Carlo error bars) are calculated by $10^{5}$ random insertions of a molecule at $300 \mathrm{~K}$ using a rigid framework and a classical force field. ${ }^{33}$ The fitted curve (green) is a sixth-order polynomial in $\tan \delta$ (eq 7). The vertical lines (red) indicate the shape of the experimentally observed $\mathrm{lp}$ and $\mathrm{np}$ structures.

molecule is assumed to interact by an equal amount $\Delta U$ which depends on the shape of the host: $F_{\text {int }}=N \Delta U$. A sixth-order polynomial in $\tan \delta$ is proposed.

$$
\begin{aligned}
& F_{\text {int }}(N, \delta, T)=N \Delta U(\delta, T)=N\left(c_{0}+c_{1} \tan \delta\right. \\
& \quad+c_{2} \tan ^{2} \delta+c_{3} \tan ^{3} \delta+c_{4} \tan ^{4} \delta+c_{5} \tan ^{5} \delta \\
& \left.\quad+c_{6} \tan ^{6} \delta\right)
\end{aligned}
$$

The coefficients $c_{i}$ depend on the temperature and the type of molecules, with $c_{6}>0$ to ensure divergence when the structure collapses $(\delta \rightarrow \pm \pi / 2)$.

The interaction energy of a single $\mathrm{CO}_{2}$ molecule has been derived from Monte Carlo simulations for a range of shapes by tracing the energy change $\Delta U_{i}$ due to random insertions of a $\mathrm{CO}_{2}$ or $\mathrm{CH}_{4}$ molecule in the unit cell. ${ }^{56,57}$ The probe molecule is inserted (and immediately removed) repeatedly at random positions in the empty framework for $N_{\text {trial }}$ attempts, and the average energy is calculated as $\sum_{i}^{\text {Ntrial }} \Delta U_{i} \exp \left(-\Delta U_{i} k_{\mathrm{B}} T\right) / N_{\text {trial }}$ where $\Delta U_{i}$ is determined via the Lennard-Jones potential (details in S.I.). The coefficients in the above equation are fitted to the calculated data points and are reported in Table 1. Figure 6 presents the Monte Carlo data with their error bar and the fitted polynomials for both the $\mathrm{CO}_{2}$ and $\mathrm{CH}_{4}$ molecule. The $\Delta U$ Monte Carlo curve of $\mathrm{CO}_{2}$ shows a value of $-20.5 \mathrm{~kJ} / \mathrm{mol}$ for the lp shape, while the adsorption of $\mathrm{CO}_{2}$ in the np shape is

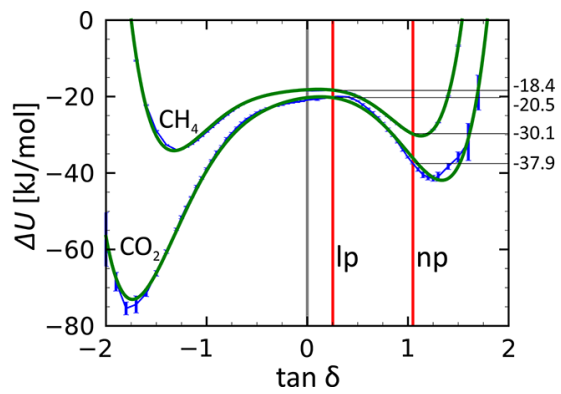

Figure 6. Average adsorption energy $\Delta U[\mathrm{~kJ} / \mathrm{mol}]$ for $\mathrm{CO}_{2}$ and $\mathrm{CH}_{4}$ in MIL-53(Cr) as a function of the shape. Monte Carlo data (blue, with Monte Carlo error bars) are calculated by $10^{5}$ random insertions of a molecule at $300 \mathrm{~K}$ using a rigid framework and a classical force field. ${ }^{33}$ The fitted curve (green) is a sixth-order polynomial in $\tan \delta$ (eq 8 ). The vertical lines (red) indicate the shape of the experimentally observed $\mathrm{lp}$ and $\mathrm{np}$ structures. 
Legend

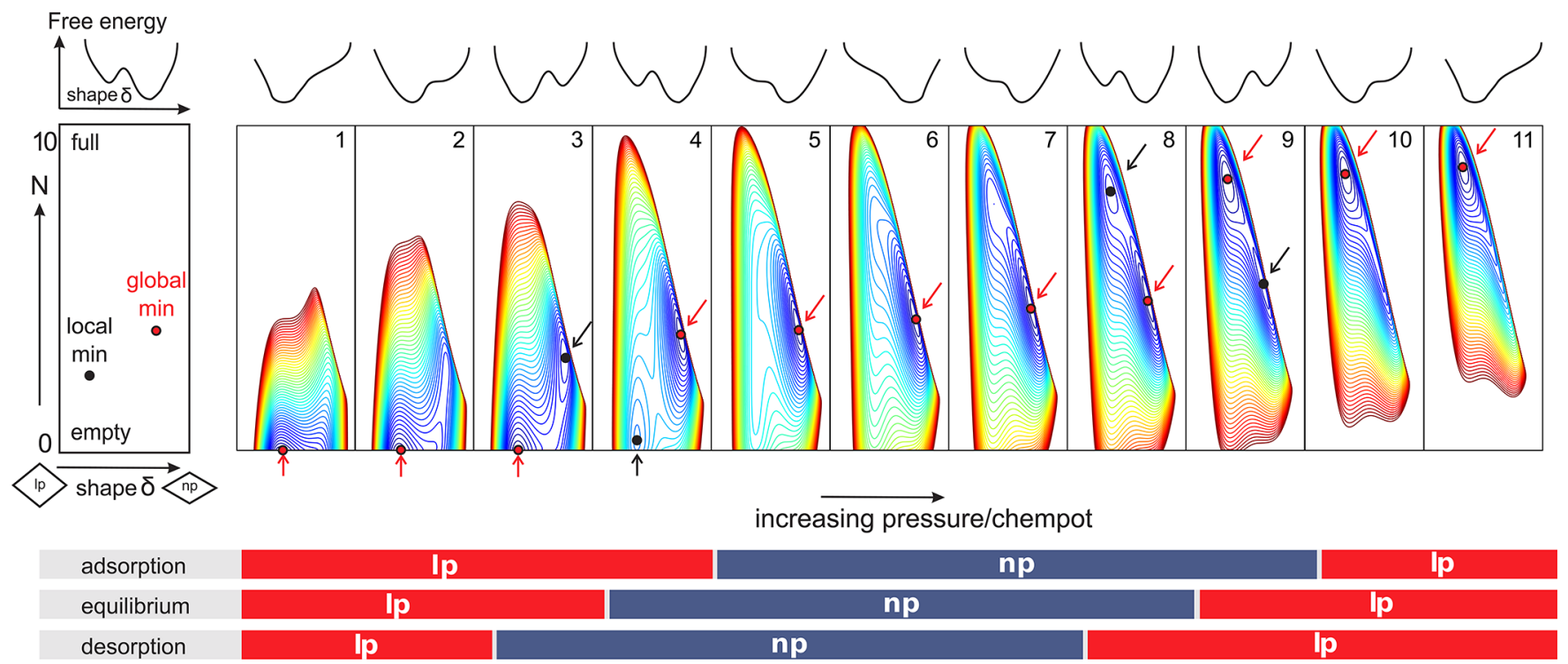

Figure 7. Free energy contours of $F+P V-N \mu$ (F in eq 3) of $\mathrm{CO}_{2}$ adsorption in MIL-53(Cr) at $300 \mathrm{~K}$ are shown for a series of pressures. In each subplot the minimum (eq 8) is indicated with a red dot and a possible second minimum with a black dot; arrows are pointing to the minima for clarity. Coloring goes from blue for the lowest free energy to red for higher values, covering a range of $80 \mathrm{~kJ} / \mathrm{mol}$ in steps of $2 \mathrm{~kJ} / \mathrm{mol}$. Above: schematic free energy profiles. The barrier in subplots 3-4 and 8-9 is responsible for the hysteresis. Below: expected phase in adsorption, equilibrium, and desorption process.

much more favorable with an adsorption energy of $-37.9 \mathrm{~kJ} /$ $\mathrm{mol}(-37.5 \mathrm{~kJ} / \mathrm{mol}$ for the experimentally observed $\mathrm{np}$ structure). Clearly, the interaction of a carbon dioxide molecule with one pore wall (benzene linker) is favorable, and the interaction with two pore walls-which is the case in the np shape-is approximately twice as favorable. Methane is found to be less adsorbed by MIL-53. $\Delta U$ is slightly less negative in the lp phase with a value $-18.4 \mathrm{~kJ} / \mathrm{mol}$ and considerably less attractive in the $\mathrm{np}$ phase with a value $-30.1 \mathrm{~kJ} / \mathrm{mol}$, which is in fair agreement with the experimental value of $-17 \mathrm{~kJ} / \mathrm{mol}$ in the lp phase. ${ }^{58}$ Such a difference has previously been noted experimentally in the adsorption enthalpy as well. ${ }^{50}$ Adsorption also seems favorable for "overstretched np" shapes, i.e., $\tan \delta<$ -1 . However, such overstretched shapes only exist theoretically because MIL-53's free energy profile $F_{\text {host }}$ rapidly increases once $\delta$ falls below $0^{\circ}$, and even the favorable $\mathrm{CO}_{2}$ interactions cannot stabilize the overstretched shapes.

F. Legendre Transform to a Constant $(n, \mu, P, T)$ Ensemble. The transition from the constant $(n, N, V(\delta), T)$ ensemble to an ensemble that is more closely related to the experimental setup, i.e., the constant $(n, \mu, P, T)$ ensemble with constant external chemical potential $\mu$ and pressure $P$, is realized by a Legendre transformation with respect to the variables $N$ and $\delta$, yielding the osmotic thermodynamic potential $X$

$$
X(n, \mu, P, T)=\min _{N, \delta}\left[F_{\text {host }}+F_{N}+F_{\text {int }}-N \mu+P V(\delta)\right]
$$

The more general case of six variable unit cell parameters is based on a Legendre transform from the constant $(n, N, \overline{\bar{\eta}}, T)$ ensemble to the constant $(n, \mu, \overline{\bar{\sigma}}, T)$ ensemble, with constant external chemical potential $\mu$ and stress tensor $\overline{\bar{\sigma}}$. Nevertheless, the general transform simplifies to the familiar $-N \mu+P V$ transform in the case of isotropic pressure (i.e., $\sigma_{i j}=-P \delta_{i j}$ ) and the use of one shape parameter (i.e., $\delta$ ) instead of all six parameters, as is derived in detail in the S.I.

In section IV, our model for the free energy in eq 8 is employed to predict the phase behavior of MIL-53(Cr) in the following way. For a given $\mu$ and $P$, the free energy $F_{\text {host }}+F_{N}+$ $F_{\text {int }}-N \mu+P V(\delta)$ represents a surface in the variables $N$ and $\delta$. The thermodynamically stable states of the system are the values $(N, \delta)$ for which the free energy reaches a minimum. In practice, free energy contours are calculated on a $(N, \delta)$ grid, and the minima are located by inspecting the free energy contour plots. The plot of the stable $(N, \delta)$ as a function of the external pressure and chemical potential represents the $(\mu, P)$ phase diagram at constant $T$. As will be shown in section IV, for some pressures two stable states exists, which is the origin for the coexistence of and/or hysteresis between the phases.

G. Comparison of the Here-Proposed Model with Earlier Work. It is instructive to compare our approach with the theory of Coudert et al. On the basis of a mean-field approach for the host atom positions, Coudert et al. approximate this ensemble with a pseudo-osmotic ensemble, by considering the osmotic potentials of the two phases separately, $\Omega_{\mathrm{os}}^{\mathrm{lp}}$ and $\Omega_{\mathrm{os}}^{\mathrm{np}} \cdot{ }^{38,47,48}$ The osmotic potentials of the phases were calculated by integration of hypothetical Langmuir isotherms $N^{\mathrm{lp}}(\mu)$ and $N^{\mathrm{np}}(\mu)$ in the lp and $\mathrm{np}$ phase, respectively. ${ }^{38}$ The difference $\Delta \Omega_{\mathrm{os}}=\Omega_{\mathrm{os}}^{\mathrm{lp}}-\Omega_{\mathrm{os}}^{\mathrm{np}}$ was successfully used to predict the transitions (none, one, or two) between the $\mathrm{lp}$ and $\mathrm{np}$ phase.

Here, in this paper, we refrain from splitting up the osmotic potential into two potentials for the phases separately. Instead, the free energy is assumed to incorporate all possible volumes, including the $\mathrm{lp}$ and $\mathrm{np}$ volumes but also all intermediate volumes. It is then not the difference $\Delta \Omega_{\text {os }}$ that governs whether a transition takes place, but the complete resulting free energy surface should be inspected for the minimum free energies. The huge advantage is that our approach can detect the existence of multiple minima, which indicates that phases 

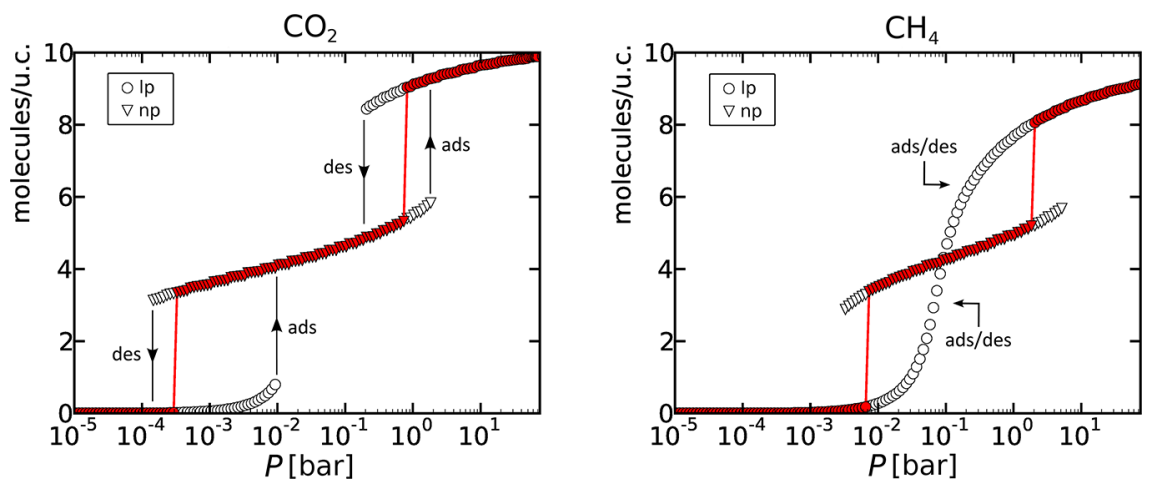

Figure 8. Calculated isotherms (adsorbed molecules per unit cell) for $\mathrm{CO}_{2}$ and $\mathrm{CH}_{4}$ adsorption in MIL-53(Cr) at $300 \mathrm{~K}$. Circles and triangles correspond to the $\mathrm{lp}$ and $\mathrm{np}$ phase, respectively. The solid red line connects the dots for which the shape/loading are at equilibrium. The remaining dots are the second, less stable, minima. For $\mathrm{CO}_{2}$, the phase behavior differs between the equilibrium, adsorption, and desorption process. For $\mathrm{CH}_{4}$, a switch to the np phase is only possible in equilibrium, but in the adsorption/desorption process the material stays in the lp phase due to hysteresis.

may coexist. In section IVA the (dis)appearance of the second minimum is an essential argument to explain the hysteresis between adsorption and desorption.

Miyahara et al. presented the thermodynamics of isotherm steps in jungle-gym type materials. ${ }^{45,59,60}$ In a recent insightful paper, they constructed a free energy profile of the gating transition between the open and closed shapes by combining a series of GCMC simulations with thermodynamic integration along a $3 \mathrm{D}$ reaction coordinate. ${ }^{45}$ Their model used two interpenetrated jungle-gym frameworks of rods, whose relative positions describe the accessible pore volume (without however changing the unit cell volume), and interactions were described with Lennard-Jones potentials. They concluded that the double step in the isotherm is due to a structural transition changing the pore size rather than a phase transition (condensation) of the guest molecules. Hysteresis was stated to be the consequence of a bistable free energy profile with a barrier, which may only be overcome if enough thermal energy is available. Bousquet et al. proposed a Wang-Landau based Monte Carlo sampling method to allow efficient explicit sampling of the osmotic ensemble at a given external gas pressure, which led to free energy landscapes of a simplified MIL-53 model as a function of the volume. ${ }^{43}$ The free energy profile of the empty host material is put forward to be a biparabolic potential, whose two wells' relative stability changes with gas pressure. The hysteresis loop size depends on the thermal energy needed to overcome the free energy barrier, which is currently an arbitrary threshold. Our reasoning is not based on a threshold thermal average energy but assumes a first-order phase transition (section IID). The system can only change shape by a collective transition when the barrier disappears, as if the threshold is assumed to be $0 \mathrm{~kJ} / \mathrm{mol}$.

\section{RESULTS AND DISCUSSION}

First, the free energy model is used to construct the phase behavior along the adsorption/desorption isotherm and to rationalize the origin of the hysteresis. The external chemical potential and pressure are here related by the equation of state of the adsorbing gas. Second, the complete $(\mu, P)$ phase diagram is constructed by decoupling the chemical potential and pressure. The influence of the model parameters in Table 1 is discussed, i.e., the flexibility of the host material, the interaction between the host and guest, and the size of the guest molecules.
A. Phase Transition in the $\mathrm{CO}_{2}$ and $\mathrm{CH}_{4}$ Adsorption Experiment. In a standard isotherm measurement, the gas pressure in the chamber is gradually increased. Consequently, the chemical potential of the gas also increases according to its equation of state. Figure 7 presents a series of free energy surfaces of $\mathrm{CO}_{2}$ adsorption in MIL-53(Cr) at $300 \mathrm{~K}$ for different pressures, calculated with the free energy expression of $X(n, \mu, P, T)$ in eq 8 where the equation of state $P=P(\mu, T)$ holds. Each subplot is a free energy contour plot with the shape $(\delta)$ and the number of molecules per unit cell $(N)$ on the axes. The global minimum and a possible second minimum are indicated with a dot. At low pressure, the unit cell is almost empty (subplot 1). There is a single minimum for the lp shape with less than one adsorbed molecule per unit cell, as imposed by the $F_{\text {host }}$ profile. With increasing pressure, a second minimum is created at the np shape with approximately 3.6 adsorbed molecules per unit cell (subplot 2). The free energy of the $\mathrm{np}$ phase decreases with increasing pressure, thus stabilizing the $\mathrm{np}$ phase (subplot 3 ). For a given transition pressure $P_{\mathrm{t}}$, the free energy of the np becomes lower than the lp phase, making $n p$ the most stable phase (subplot 4), and for a given, even higher pressure, the minimum at the lp phase disappears (subplot 5), such that the system surely takes the $n p$ shape (subplot 6). Next, the reverse phenomenon occurs at a higher transition pressure $P_{\mathrm{t}}^{\prime}$. The minimum at the lp shape, however, reappears with a higher number of molecules (subplot 7) and gradually stabilizes (subplot 8) until it becomes the most stable phase (subplot 9). The minimum at the np shape becomes less stable until it disappears (subplot 10), and the lp shape is the only phase that remains at high pressure (subplot 11).

Our calculations thus confirm that the np shape can be stabilized by interactions of the adsorbing $\mathrm{CO}_{2}$ molecules even if the $n p$ shape is not a stable phase for the empty host material at the given temperature ( $F_{\text {host }}$ has only a minimum in the lp shape in the model). Since the $\mathrm{CO}_{2}$ preferably adsorbs in the $\mathrm{np}$ shape, the guest material tends to shrink as soon as the number of molecules is high enough to overcome the resistance by the host material (flexibility parameters $k_{i}$ figuring in $F_{\text {host }}$ ). The material should be flexible enough and the host-guest interaction strong enough to see this $\mathrm{lp} \rightarrow \mathrm{np}$ transition. A different effect is responsible for the opening of the structure at higher pressures. Once the narrow pores are fully filled with molecules, the structure needs to transform to the lp shape to 

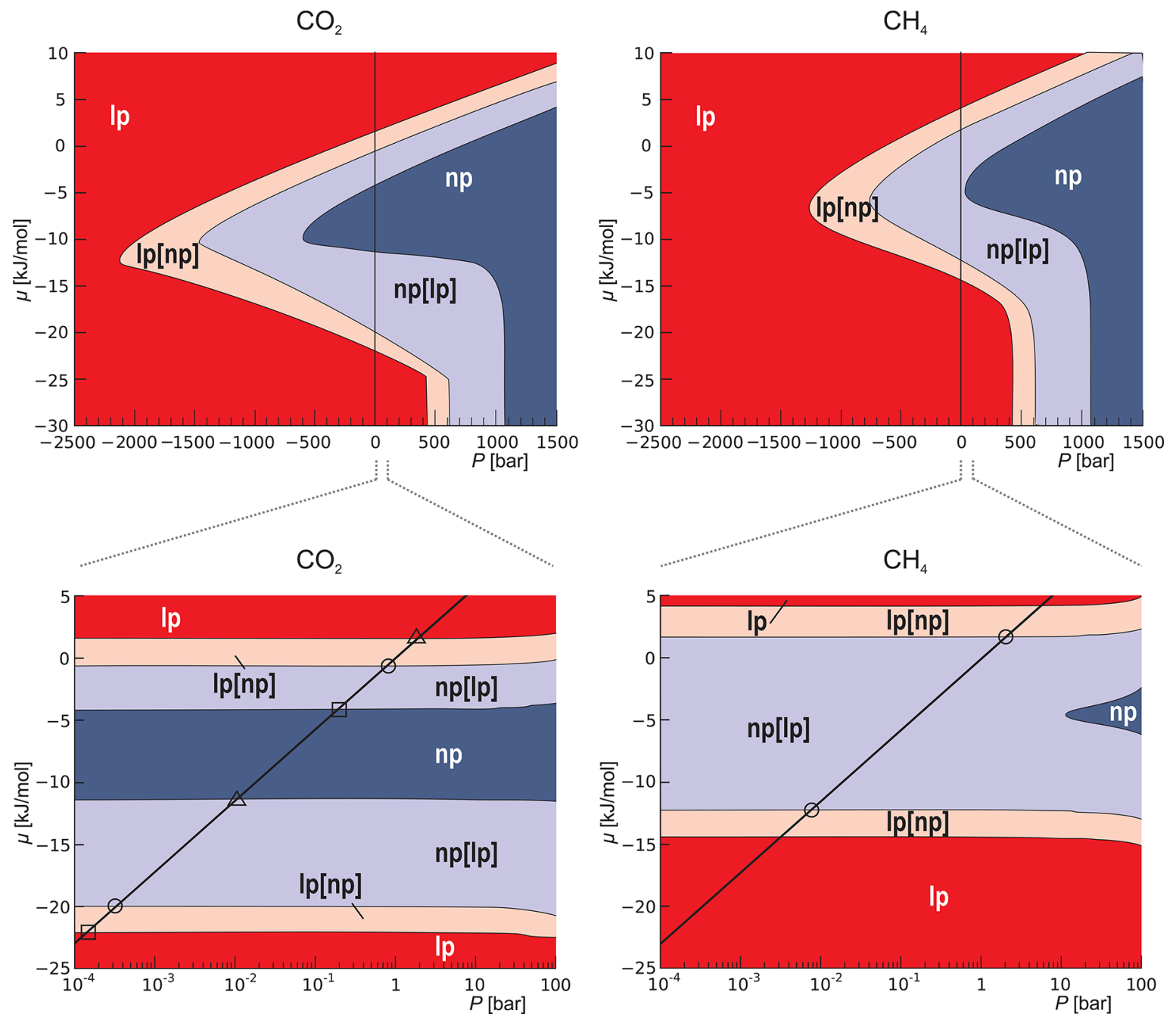

Figure 9. $(\mu, P)$ phase diagram of $\mathrm{CO}_{2}$ and $\mathrm{CH}_{4}$ adsorbed in MIL-53(Cr) at $300 \mathrm{~K}$. Some regions are the pure lp or pure np phase (label lp or np), while in intermediate regions coexistence between the $\mathrm{lp}$ and $\mathrm{np}$ is possible (label $\mathrm{lp}[\mathrm{np}]$ if lp is most stable phase or label $\mathrm{np}[\mathrm{lp}]$ if $\mathrm{np}$ is most stable phase). Chemical potential [in $\mathrm{kJ} / \mathrm{mol}$ ] is expressed with respect to the reference potential $\mu_{0}$ of an ideal gas at pressure $P_{0}=1$ bar and $T=300 \mathrm{~K}$. (Top) The phase behavior for negative pressures has also been calculated. (Bottom) Inset on the typical experimental pressure range (logarithmic pressure scale). The equation of state (black line) intersects with the $\mathrm{np}$ region for $\mathrm{CO}_{2}$ but not for $\mathrm{CH}_{4}$. The transition pressures at which the lp $\leftrightarrow$ np transitions take place are indicated in the case of an equilibrium (circles), adsorption (triangles), and desorption (squares) process.

accommodate additional molecules. The larger the size of the molecules, the sooner the transition to the large pore occurs.

Experimentally, the transitions $\mathrm{lp} \leftrightarrow \mathrm{np}$ and $\mathrm{np} \leftrightarrow \mathrm{lp}$ occur at lower pressure in the desorption (decreasing $P$ ) than in the adsorption (increasing $P$ ) isotherm measurement. This hysteresis effect can also be explained by the (dis)appearance and (de)stabilization of the minima. For this purpose, schematic free energy profiles were plotted above each free energy contour subplot (Figure 7) to show the relative stability of the $\mathrm{lp}$ and $\mathrm{np}$ phase. When the global equilibrium is attained, the structure would always take the phase corresponding to the global minimum. However, in reality the structure experiences a free energy barrier between the minima. In the gas phase, transitions may be thermally activated such that a barrier of the order of $k_{\mathrm{B}} T$ can be overcome in a reasonably short time. In contrast, in the solid state all unit cells should simultaneously change their shape, converting a barrier of $k_{\mathrm{B}} T$ per unit cell into an enormous barrier for the entire crystal. As a consequence, a transition from one minimum to another can only take place when the barrier disappears, i.e., when that first minimum disappears! This happens in subplots 5 and 10 in the adsorption measurement (higher pressures than equilibrium situation) and in subplots 2 and 7 in the desorption measurement (lower pressures than equilibrium situation), thus explaining the hysteresis between adsorption and desorption. Our explanation differs from the mechanism proposed by Triguero et al. where the hysteresis evolves from a coupling term between the $\mathrm{lp}$ and $\mathrm{np}$ phases, which are assumed to be both present in inhomogeneous crystals. ${ }^{40}$

The adsorption isotherm at $300 \mathrm{~K}$ can be obtained by tracing the predicted number of adsorbed molecules in the stable states as a function of the pressure. Figure 8 shows the adsorbed amount of $\mathrm{CO}_{2}$ or $\mathrm{CH}_{4}$ in the case of an equilibrium (red line), adsorption, and desorption process. For each stable state, not only the adsorbed amount is available but also information about the shape is known. In Figure 8 the circles and diamond symbols correspond to the lp and np phase, respectively. The red line connects the most stable states, which is followed in the case global equilibrium could be attained. The remaining dots are the second minima which are less stable. In the adsorption and desorption measurement, our interpretation of the hysteresis prescribes that a transition to a different phase only 
(a) more flexible $\left(0.8 F_{\text {host }}\right)$

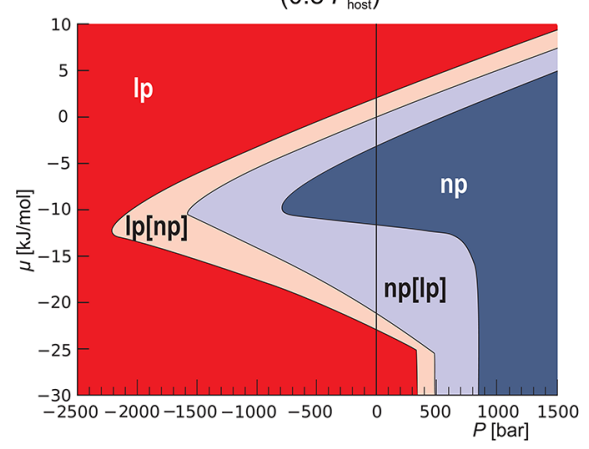

(b) more rigid $\left(1.2 F_{\text {host }}\right)$

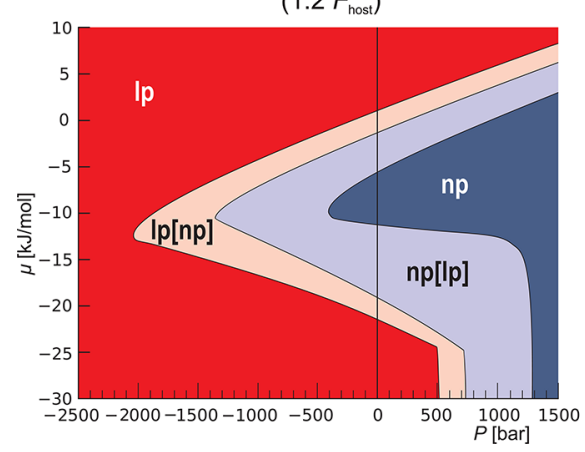

(c) less interaction $(0.8 \Delta U)$

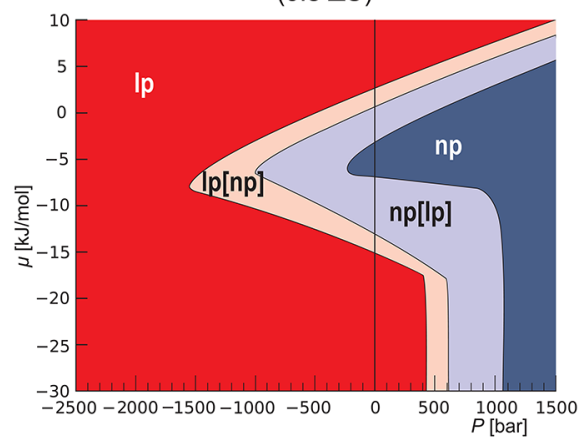

(d) more interaction $(1.2 \Delta U)$

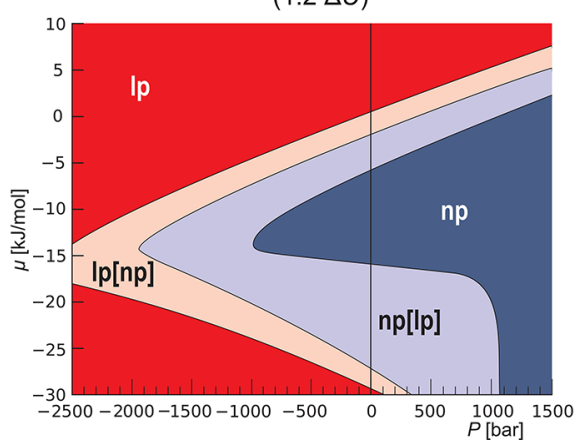

(e) smaller molecules $(0.8 b)$

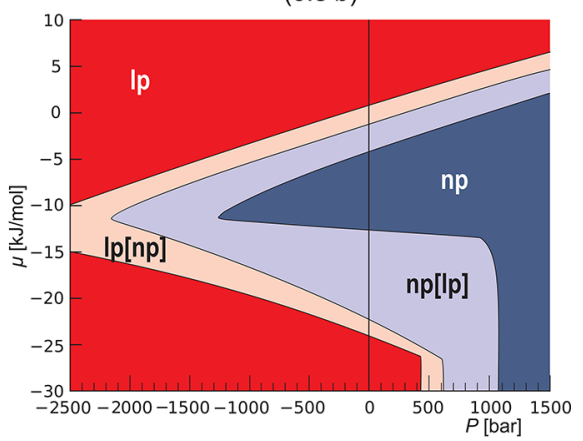

(f) larger molecules $(1.2 b)$

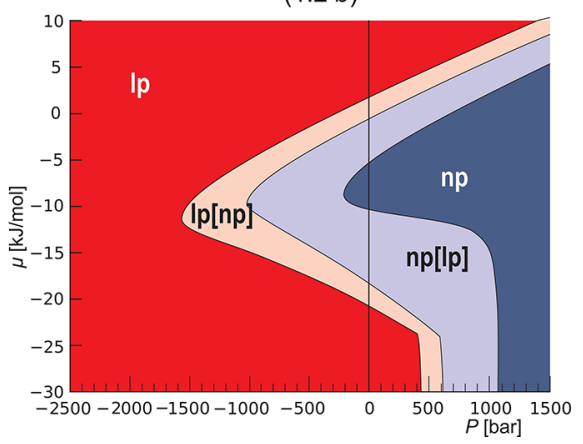

Figure 10. Influence of framework flexibility $\left(F_{\text {host }}\right)$, host-guest interaction $(\Delta U)$, and molecule size ( $b$ van der Waals) on the phase diagram of MIL-53(Cr). The starting point is the phase diagram of $\mathrm{CO}_{2}$ at $300 \mathrm{~K}$ in Figure 9. The different terms are one by one rescaled to simulate a difference of $\pm 20 \%$. (a) $0.8 F_{\text {host }}$; (b) $1.2 F_{\text {host }}$ (c) $0.8 \Delta U$; (d) $1.2 \Delta U$; (e) $0.8 b$; and (f) $1.2 b$. Chemical potential [in $\mathrm{kJ} / \mathrm{mol}$ ] is expressed with respect to the reference potential $\mu_{0}$ of an ideal gas at pressure $P_{0}=1$ bar and $T=300 \mathrm{~K}$.

takes place when the stable state at the current phase disappears. In other words, the system remains in a stable state as long as possible, even when this state is no longer the most stable one. Therefore, the transitions are postponed to a higher and lower pressure, for the adsorption or desorption isotherm, respectively. As a result, for $\mathrm{CO}_{2}$, the phase behavior differs between the equilibrium, adsorption, and desorption process. For $\mathrm{CH}_{4}$, a switch to the np phase would only be possible in an infinitely slowly equilibrium process, but in the adsorption/desorption process, the material always remains in the lp phase due to the collective behavior.

B. $(\mu, P)$ Phase Diagram. Phase Diagram of Carbon Dioxide Adsorption. The external chemical potential and pressure act as two independent thermodynamic variables in the osmotic ensemble. Even if in experimental setups this decoupling is hard to realize, our free energy model is perfectly suited to investigate the phase behavior in the complete $(\mu, P)$ phase diagram from a theoretical point of view. This allows us, in principle, to predict how changing the thermodynamic control variables may induce breathing. It is also possible to consider negative pressures, which may allow us to design new applications for this breathing material since two decoupled control variables are at hand instead of only one.

For a range of combinations $(\mu, P)$ of chemical potentials and pressures the minima of the free energy surface are inspected, and the phase is determined. The resulting $(\mu, P)$ phase diagram is split into four regions. In the lp or np regions only the lp phase or the np phase is stable, respectively. In the intermediate regions $\mathrm{lp}[\mathrm{np}]$ and $\mathrm{np}[\mathrm{lp}]$ there is coexistence between the two phases: in lp[np] the large-pore phase is the most stable with a metastable narrow-pore phase, while in $\mathrm{np}[\mathrm{lp}]$ it is the opposite. A single crystal can only take either the lp or $\mathrm{np}$ phase due to geometrical constraints, so the coexistence means that a powder consists of lp crystals and np crystals. Figure 9 shows the resulting phase diagram of MIL$53(\mathrm{Cr})$ upon adsorption of $\mathrm{CO}_{2}$ molecules. It is clear that a np region surrounded by a lp region is present; this is the first condition for occurrence of the breathing effect. Moreover the equation of state $\mu(P, T)$ of the $\mathrm{CO}_{2}$ gas cuts through this $\mathrm{np}$ region, such that also the second condition for breathing is satisfied. Interestingly, the $(\mu, P)$ phase diagram largely resembles the hypothetical diagram (Figure 3 ) as discussed in section II. In conclusion, our model successfully predicts the breathing of MIL-53(Cr) under adsorption of $\mathrm{CO}_{2}$.

The van der Waals equation of state line $\mu(P, T)$ cuts through all four different regions, including the coexistence regions, in the pressure range $10^{-4}$ to 1 bar (logarithmic plot in Figure 9). The logarithmic plot in Figure 9 zooms on the low pressure range. The frontiers of the regions are fairly horizontal because the $P V$ term merely takes a value of about $0.1 \mathrm{~kJ} / \mathrm{mol}$ at $P=1$ bar. This indicates that adding low mechanical pressure has little effect-over 100 bar is needed to induce structural changes by changing pressure. Structural changes in this pressure range should be induced by changing the chemical potential. The transition pressures $P_{\mathrm{t}}$ and $P_{\mathrm{t}}^{\prime}$ can be read from the phase diagram. The equilibrium pressures at which the transitions take place lie on the border between the $\operatorname{lp}[\mathrm{np}]$ and $\mathrm{np}[\mathrm{lp}]$ region, assuming the lowest free energy minimum is always selected. The phase transition $1 \mathrm{p} \leftrightarrow \mathrm{np}$ takes place at a transition pressure $P_{\mathrm{t}} \approx 5 \times 10^{-4}$ bar and the transition $\mathrm{np} \leftrightarrow \mathrm{lp}$ at $P_{\mathrm{t}}^{\prime} \approx 0.5$ bar. In an adsorption experiment, the phase transitions $\mathrm{lp} \rightarrow \mathrm{np}$ and $\mathrm{np} \rightarrow \mathrm{lp}$ occur on the border $\mathrm{np}[\mathrm{lp}] \rightarrow \mathrm{np}$ 
and the border $\operatorname{lp}[\mathrm{np}] \rightarrow \mathrm{lp}$, when the $\mathrm{lp}$ and the $\mathrm{np}$ phase disappear, respectively. The corresponding two transition pressures are indicated on the diagram (Figure 9). Similarly, the transition pressures in the desorption process are located on the $\mathrm{np}[\mathrm{lp}] \rightarrow \mathrm{np}$ and $\mathrm{lp}[\mathrm{np}] \rightarrow \mathrm{lp}$ borders. The calculated numerical values of the transition pressures lie below the experimental values. On one hand the experimental setup is prone to errors for instance because of imperfections of the crystal fabrication. On the other hand the separation into three parts (eq 3 ) and the van der Waals equation are probably too rude approximations to achieve quantitative accuracy. Nevertheless, the qualitative picture obtained with our free energy model is very satisfactory.

Phase Diagram of Methane Adsorption. We have also derived a free energy model for methane following the same procedure as in section III. With respect to $\mathrm{CO}_{2}$ few parameters need to be changed: the van der Waals parameters $a$ and $b$ can be taken from the literature, while the host-guest interaction $\Delta U$ is obtained from Monte Carlo simulations with methane Widom insertions at $300 \mathrm{~K}$ (Figure 6, Table 1). The van der Waals parameters of $\mathrm{CH}_{4}$ are similar to those of $\mathrm{CO}_{2}$; however, the interaction profile differs, probably because methane is an apolar molecule without quadrupole moment, while carbon dioxide has a high quadrupole moment. In our Monte Carlo Widom insertions in the empty host, the adsorption energy of $\mathrm{CH}_{4}$ in the lp shape is in absolute value slightly smaller than for $\mathrm{CO}_{2}$; in the np shape it is $7.8 \mathrm{~kJ} / \mathrm{mol}$ less attractive than for $\mathrm{CO}_{2}$ (Figure 6). These differences with respect to $\mathrm{CO}_{2}$ shift the $\mathrm{np}$ region in the methane phase diagram upward and to the right (Figure 9). Consequently, the equation of state $\mu(P, T)$ barely cuts through the $\mathrm{np}$ region, and the breathing effect is expected to be very limited, as illustrated in Figure 8. Indeed, $\mathrm{CH}_{4}$ adsorption has not been observed to cause breathing in isotherm experiments. ${ }^{30}$

The ratio of the lp versus np Henry coefficients, which are closely related to the adsorption energies, have been interpreted as the key quantity determining the number of structural transitions. ${ }^{48}$ A similar conclusion is found in our model: the adsorption of the gas molecules in the np phase has to be favorable enough compared to the lp phase to cause structural transitions; otherwise, the framework's limited flexibility prevents it from taking the narrow shape.

Influence of Framework Flexibility, Host-Guest Interaction, and Molecule Size. In this section we investigate the response of the phase diagram to changes of the various model parameters. In particular the influence is studied of the host flexibility $F_{\text {host }}$ the host-guest interaction $\Delta U$, and the molecule size by means of the van der Waals parameter $b$. The diagrams correspond with hypothetical systems but are nevertheless useful to explore the importance and effect of each parameter. The reference diagram is the $\mathrm{CO}_{2}$ adsorption in MIL-53(Cr). Figure 10 shows the diagrams when imposing deviations of $\pm 20 \%$ with respect to the reference parameters.

First, multiplying the host free energy $F_{\text {host }}$ with a factor of 0.8 makes the framework 20\% more flexible. In Figure 10a, the entire phase diagram is shifted to the left. In the right bottom of the diagram the transition $\mathrm{l} \rightarrow \mathrm{np}$ takes place at lower pressure, consistent with the idea of a more flexible (rigid) framework. Even if the size of the $n p$ region remains the same, the intersection with the equation of state becomes larger when shifted to the left, which means that the np phase is present over a larger pressure range in the isotherm experiment. Indeed, a very flexible framework is expected to transition to its $n p$ shape easily. Multiplying $F_{\text {host }}$ with a factor of 1.2 makes the framework more rigid. The phase diagram shifts to the right in Figure $10 \mathrm{~b}$, and the transition of the $\mathrm{lp}$ to the $\mathrm{np}$ phase takes place at higher pressure.

Second, multiplying the host-guest interaction $\Delta U$ with a factor 0.8 shifts the np region to the right and upward (Figure $10 \mathrm{c})$, indicating that more pressure or a higher number of adsorbed molecules is required to close the structure. Since the average adsorption energy per molecule is lower, it takes more adsorbed molecules to overcome the framework resistance. Moreover, the np region shrinks in size such that the equation of state curve barely intersects the np region. The occurrence of breathing is less probable with molecules that have a weak interaction with the host material. Increasing the interaction by multiplying $\Delta U$ with 1.2 shifts the $\mathrm{np}$ region to the left and downward (Figure 10d). The size of the np region increases considerably. Apparently the structure easily closes because of the favorable interactions in the np phase. The structure fills up more quickly than in the reference diagram such that it opens at a lower pressure. Thus, a gas with relatively strong host-guest interaction is a likely candidate molecule to induce breathing in flexible materials. However, breathing may still be prohibited despite the strong interactions. A first reason is when the interaction is extremely strong: the lp phase at the bottom of the diagram is reached only with extremely low pressures, which might be experimentally unachievable. A second reason is when the interaction profile differs qualitatively from the one of $\mathrm{CO}_{2}$ and a preferable adsorption in the $\mathrm{np}$ over the lp phase is lacking, such that the resulting phase diagram might look differently alltogether.

Third, multiplication of the $b$ van der Waals parameter in $F_{N}$ with 0.8 decreases the size of the gas molecules. A particular value for the chemical potential $\mu$ corresponds to a higher particle density for the smaller molecules than for the reference molecules. A higher particle density also implies a higher amount of adsorbed molecules since the energetics (parameter $a$ in $\left.F_{\text {host }} \Delta U\right)$ remained untouched. More small molecules fit into the narrow pore. Each molecule contributes to the stabilization of the np phase by an amount $\Delta U$ such that the np region is more prominent in the diagram. Consequently, the np region shifts to the left and is elongated (Figure 10e). In contrast, multiplication of $b$ with 1.2 decreases the size of the $\mathrm{np}$ region (Figure 10f). With larger molecules there might not be enough space in the narrow pore to adsorb all molecules needed to overcome the resistance of the framework, thus suppressing breathing.

\section{CONCLUSION}

The chemical potential and the pressure are two independent thermodynamic variables which in most experimental setups are related by an equation of state. By removing this constraint, we have proposed a complete $(\mu, P)$ phase diagram for flexible porous materials. A free energy model has been developed which incorporates the key factors to describe the thermodynamics of the breathing phenomenon: (1) the resistance of the host material to deformations, i.e., the empty host flexibility, (2) the host-guest interaction, where breathing requires a more favorable adsorption in the $\mathrm{np}$ compared to the $\mathrm{lp}$ phase to induce the closing of the structure, and (3) the fact that pores can be "full", which initiates the reopening of the structure to accommodate more molecules with increasing pressure. The parameters of this free energy model are fitted based on a combination of experimental and simulated Monte Carlo data. 
By inspection of the minima of the free energy, the phase behavior of MIL-53( Cr) during a $\mathrm{CO}_{2}$ isotherm experiment is explained. With increasing pressure, a second minimum is created, stabilizes, destabilizes, and disappears due to the balance between the mentioned thermodynamical key factors. Moreover, the free energy contours nicely show the thermodynamic cause of the hysteresis effect, when it is assumed that all unit cells of a crystal need to transform simultaneously. Further investigation is however needed to support our hypothesis of collective behavior and to construct reliable free energy profiles of the empty host at finite temperature.

Despite its simpleness, our free energy model succeeds in discriminating between the phase behavior upon $\mathrm{CO}_{2}$ and $\mathrm{CH}_{4}$ adsorption in MIL-53(Cr) at $300 \mathrm{~K}$. A more detailed study of the model parameters gives insight into their influence. If the adsorption energy profile as a function of the shape is similar to the one of $\mathrm{CO}_{2}$, the breathing effect is expected to be more prominent for more flexible materials, when adsorbate molecules have stronger host-guest interaction, and for smaller adsorbate molecules.

The methodology is not only valid for MIL-53(Cr) but also generic and should in principle work for a broader range of breathing materials. The model is expected to perform well when no adsorption sites with very different strength occur in the pore. In the presence of distinct adsorption sites, the homogeneous description of the adsorbed gas molecules with a van der Waals equation of state and the same average adsorption energy per molecule might fail, and an extension of the model might be required. The first logical extension would be to test the new model on a series of MIL-53 materials with other metals at the nodal points and simulate whether breathing is observed. The free energy profile of the empty host material would need to be recalculated, as changing a metal influences the flexibility of a material. ${ }^{55}$ The interaction profile would also need to be recalculated using another metal at the chromium position. Our methodology can also be applied on structures with MIL-53 topology but longer or functionalized linkers (e.g., MIL-53- $\mathrm{NH}_{2},{ }^{10,28} \mathrm{COMOC}^{4 a m i l y}{ }^{61}$ ), on other MOF families (e.g., DMOF-1 ${ }^{42}$ ), or on adsorption with other gases can be investigated.

\section{ASSOCIATED CONTENT}

\section{S Supporting Information}

Structural parameters for MIL-53(Cr), free energy profile of empty host, pore volume and host-guest interaction calculations, force field parameters, and legendre transform with respect to volume. This material is available free of charge via the Internet at http://pubs.acs.org.

\section{AUTHOR INFORMATION}

\section{Corresponding Author}

*E-mail: an.ghysels@ugent.be.

\section{Notes}

The authors declare no competing financial interest.

\section{ACKNOWLEDGMENTS}

This work was supported by the Fund for Scientific Research Flanders (FWO), the research Board of Ghent University, and BELSPO in the frame of IAP 7/05. Funding was also received from the European Research Council under FP7 with ERC grant agreement number 240483. Computational resources and services were provided by Ghent University (Stevin Supercomputer Infrastructure). A.G. is a Post-Doctoral Fellow with the FWO and received a grant of the Fulbright Commission for Educational Exchange. L.V. is Aspirant Fellow with the FWO. B.S. was supported as part of the Center for Gas Separations Relevant to Clean Energy Technologies, an Energy Frontier Research Center funded by the U.S. Department of Energy, Office of Science, Office of Basic Energy Sciences under Award Number DE-SC0001015.

\section{REFERENCES}

(1) Long, J. R.; Yaghi, O. M. The Pervasive Chemistry of MetalOrganic Frameworks. Chem. Soc. Rev. 2009, 38, 1213-1214.

(2) Eddaoudi, M.; Kim, J.; Rosi, N.; Vodak, D.; Wachter, J.; O’Keeffe, M.; Yaghi, O. M. Systematic Design of Pore Size and Functionality in Isoreticular MOFs and Their Application in Methane Storage. Science 2002, 295, 469-472.

(3) Yaghi, O. M.; O'Keeffe, M.; Ockwig, N. W.; Chae, H. K.; Eddaoudi, M.; Kim, J. Reticular Synthesis and the Design of New Materials. Nature 2003, 423, 705-714.

(4) Férey, G. Hybrid Porous Solids: Past, Present, Future. Chem. Soc. Rev. 2008, 37, 191-214.

(5) Tranchemontagne, D. J.; Mendoza-Cortes, J. L.; O'Keeffe, M.; Yaghi, O. M. Secondary Building Units, Nets and Bonding in the Chemistry of Metal-Organic Frameworks. Chem. Soc. Rev. 2009, 38, 1257-1283.

(6) Perry, J. J.; Perman, J. A.; Zaworotko, M. Design and Synthesis of Metal-Organic Frameworks using Metal-Organic Polyhedra as Supermolecular Building Blocks. Chem. Soc. Rev. 2009, 38, 1400-1417.

(7) Horike, S.; Shimomura, S.; Kitagawa, S. Soft Porous Crystals. Nature Chem. 2009, 1, 695-704.

(8) Férey, G.; Serre, C.; Devic, T.; Maurin, G.; Jobic, H.; Llewellyn, P. L.; De Weireld, G.; Vimont, A.; Daturi, M.; Chang, J. S. Why Hybrid Porous Solids Capture Greenhouse Gases? Chem. Soc. Rev. 2011, 40, $550-562$.

(9) D’Alessandro, D. M.; Smit, B.; Long, J. R. Carbon Dioxide Capture: Prospects for New Materials. Angew. Chem., Int. Ed. 2010, 49, 6058-6082.

(10) Couck, S.; Denayer, J. F. M.; Baron, G. V.; Remy, T.; Gascon, J.; Kapteijn, F. J. An Amine-Functionalized MIL-53 Metal-Organic Framework with Large Separation Power for $\mathrm{CO}_{2}$ and $\mathrm{CH}_{4}$. J. Am. Chem. Soc. 2009, 131, 6326.

(11) Finsy, V.; Ma, L.; Alaerts, L.; De Vos, D. E.; Baron, G. V.; Denayer, J. F. M. Separation of $\mathrm{CO}_{2} / \mathrm{CH}_{4}$ Mixtures with the MIL53(Al) Metal-Organic Framework. Microporous Mesoporous Mater. 2009, 120, 221-227.

(12) Alaerts, L.; Kirschhock, C. E. A.; Maes, M.; van der Veen, M. A.; Finsy, V.; Depla, A.; Martens, J. A.; Baron, G. V.; Jacobs, P. A.; Denayer, J. E. M.; De Vos, D. E. Selective Adsorption and Separation of Xylene Isomers and Ethylbenzene with the Microporous Vanadium(IV) Terephthalate MIL-47. Angew. Chem., Int. Ed. 2007, 46, 4293-4297.

(13) Alaerts, L.; Maes, M.; Giebeler, L.; Jacobs, P. A.; Martens, J. A.; Denayer, J. F. M.; Kirschhock, C. E. A.; De Vos, D. E. Selective Adsorption and Separation of ortho-Substituted Alkylaromatics with the Microporous Aluminum Terephthalate MIL-53. J. Am. Chem. Soc. 2008, 130, 14170-14178.

(14) Liu, Y.; Her, J. H.; Dailly, A.; Ramirez-Cuesta, A. J.; Neumann, D. a.; Brown, C. M. Reversible Structural Transition in MIL-53 with Large Temperature Hysteresis. J. Am. Chem. Soc. 2008, 130, 1181311818 .

(15) Beurroies, I.; Boulhout, M.; Llewellyn, P. L.; Kuchta, B.; Férey, G.; Serre, C.; Denoyel, R. Using Pressure to Provoke the Structural Transition of Metal-Organic Frameworks. Angew. Chem., Int. Ed. 2010, $49,7526-7529$.

(16) Murray, K. S.; Kepert, C. J. Cooperativity in Spin Crossover Systems: Memory, Magnetism and Microporosity. Top. Curr. Chem. 2004, 233, 195-228. 
(17) Férey, G.; Serre, C. Large Breathing Effects in ThreeDimensional Porous Hybrid Matter: Facts, Analyses, Rules and Consequences. Chem. Soc. Rev. 2009, 38, 1380-1399.

(18) Férey, G.; Mellot-Draznieks, C.; Serre, C.; Millange, F.; Dutour, J.; Surble, S.; Margiolaki, I. A Chromium Terephthalate-Based Solid with Unusually Large Pore Volumes and Surface Area. Science 2005, 309, 2040-2042.

(19) Serre, C.; Millange, F.; Thouvenot, C.; Noguès, M.; Marsolier, G.; Louër, D. A.; Férey, G. Very Large Breathing Effect in the First Nanoporous Chromium(III)-Based Solids: MIL-53 or $\mathrm{Cr}^{\mathrm{III}}(\mathrm{OH}) \cdot\left[\mathrm{O}_{2} \mathrm{C}-\mathrm{C}_{6} \mathrm{H}_{4}-\mathrm{CO}_{2}\right] \cdot\left[\mathrm{HO}_{2} \mathrm{C}-\mathrm{C}_{6} \mathrm{H}_{4}-\mathrm{CO}_{2} \mathrm{H}\right]_{x} \cdot \mathrm{H}_{2} \mathrm{O}_{y}$. J. Am. Chem. Soc. 2002, 124, 13519-13526.

(20) Millange, F.; Serre, C.; Férey, G. Synthesis, Structure Determination and Properties of MIL-53as and MIL-53ht: the First CrIII Hybrid Inorganic-Organic Microporous Solids: CrIII$(\mathrm{OH}) \cdot\left(\mathrm{O}_{2} \mathrm{C}-\mathrm{C}_{6} \mathrm{H}_{4}-\mathrm{CO}_{2}\right) \cdot\left(\mathrm{HO}_{2} \mathrm{C}-\mathrm{C}_{6} \mathrm{H}_{4}-\mathrm{CO}_{2} \mathrm{H}\right) \mathrm{x}$. Chem. Commun. 2002, 8, 822-823.

(21) Loiseau, T.; Serre, C.; Huguenard, C.; Fink, G.; Taulelle, F.; Henry, M.; Bataille, T.; Férey, G. A Rationale for the Large Breathing of the Porous Aluminum Terephthalate (MIL-53) upon Hydration. Chem.-Eur. J. 2004, 10, 1373-1382.

(22) Whitfield, T. R.; Wang, X. Q.; Liu, L. M.; Jacobson, A. J. MetalOrganic Frameworks based on Iron Oxide Octahedral Chains connected by Benzenedicarboxylate Dianions. Solid State Sci. 2005, 7, 1096-1103.

(23) Vougo-Zanda, M.; Huang, J.; Anokhina, E.; Wang, X. Q.; Jacobson, A. Tossing and Turning: Guests in the Flexible Frameworks of Metal(III) Dicarboxylates. Inorg. Chem. 2008, 47, 11535-11542.

(24) Millange, F.; Guillou, N.; Walton, R. I.; Grenèche, J.; Margiolaki, I.; Ferrey, G. Effect of the Nature of the Metal on the Breathing steps in MOFs with Dynamic Frameworks. Chem. Commun. 2008, 39, 47324734.

(25) Volkringer, C.; Loiseau, T.; Guillou, N.; Férey, G.; Elkaim, E.; Vimont, A. XRD and IR Structural Investigations of a Particular Breathing Effect in the MOF-type Gallium Terephthalate MIL53(Ga). Dalton Trans. 2009, 12, 2241-2249.

(26) Nouar, F.; Devic, T.; Chevreau, H.; Guillou, N.; Gibson, E.; Clet, G.; Daturi, M.; Vimont, A.; Greneche, J. M.; Breeze, M.; Walton, R.; Llewellyne, P.; Serre, C. Tuning the Breathing Behaviour of MIL53 by Cation Mixing. Chem. Commun. 2012, 48, 10237-10239.

(27) Biswas, S.; Ahnfeldt, T.; Stock, N. New Functionalized Flexible Al-MIL-53-X (X = -Cl, -Br, -CH3, -NO2, - $(\mathrm{OH})(2))$ Solids: Syntheses, Characterization, Sorption, and Breathing Behavior. Inorg. Chem. 2011, 50, 9518-9526.

(28) Devic, T.; et al. Functionalization in Flexible Porous Solids: Effects on the Pore Opening and the Host-Guest Interactions. J. Am. Chem. Soc. 2010, 132, 1127-1136.

(29) Serre, C.; Bourrelly, S.; Vimont, A.; Ramsahye, N. A.; Maurin, G.; Llewllyn, P. L.; Daturi, M.; Filinchuk, Y.; Leynaud, O.; Barnes, P.; Férey, G. An Explanation for the Very Large Breathing Effect of a Metal-Organic Framework during $\mathrm{CO}_{2}$ Adsorption. Adv. Mater. 2007, 19, 2246-2251.

(30) Llewellyn, P. L.; Maurin, G.; Devic, T.; Loera-Serna, S.; Rosenbach, N.; Serre, C.; Bourrelly, S.; Horcajada, P.; Filinchuk, Y.; Férey, G. Prediction of the Conditions for Breathing of Metal Organic Framework Materials Using a Combination of X-ray Powder, Diffraction, Microcalorimetry, and Molecular Simulation. J. Am. Chem. Soc. 2008, 130, 12808-12814.

(31) Kolokolov, D. I.; Jobic, H.; G., S. A.; Plazanet, M.; Zbiri, M.; Ollivier, J.; Guillerm, V.; Devic, T.; Serre, C.; Férey, G. Comparison of the Dynamics of MIL-53(Cr) and MIL-47(V) Frameworks using Neutron Scattering and DFT Methods. Eur. Phys. J. 2010, 189, 263271.

(32) Dubbeldam, D.; Krishna, R.; Snurr, R. Q. Method for Analyzing Structural Changes of Flexible Metal-Organic Frameworks Induced by Adsorbates. J. Phys. Chem. C 2009, 113, 19317-19327.

(33) Salles, F.; Ghoufi, A.; Maurin, G.; Bell, R. G.; Mellot-Draznieks, C.; Férey, G. Molecular Dynamics Simulations of breathing MOFs:
Structural Transformations of MIL-53(Cr) upon Thermal Activation and $\mathrm{CO}_{2}$ Adsorption. Angew. Chem., Int. Ed. 2008, 47, 8487-8491.

(34) Coombes, D. S.; Cora, F.; Mellot-Draznieks, C.; Bell, R. G. Sorption-Induced Breathing in the Flexible Metal Organic Framework CrMIL-53: Force-Field Simulations and Electronic Structure Analysis. J. Phys. Chem. C 2009, 113, 544-552.

(35) Walker, A. M.; Civalleri, B.; Slater, B.; Mellot-Draznieks, C.; Corà, F.; Zicovich-Wilson, C. M.; Román-Pérez, G.; Soler, J. M.; Gale, J. D. Flexibility in a Metal-Organic Framework Material Controlled by Weak Dispersion Forces: the Bistability of MIL-53(Al). Angew. Chem., Int. Ed. 2010, 49, 7501-7503.

(36) Vanduyfhuys, L.; Verstraelen, T.; Vandichel, M.; Waroquier, M.; Van Speybroeck, V. Ab Initio Parametrized Force Field for The Flexible Metal-Organic Framework MIL-53(Al). J. Chem. Theory Comput. 2012, 8, 3217-3231.

(37) Ghoufi, A.; Subercaze, A.; Ma, Q.; Yot, P. G.; Ke, Y.; PuenteOrench, I.; Devic, T.; Guillerm, V.; Zhong, C.; Serre, C.; Férey, G.; Maurin, G. Comparative Guest, Thermal, and Mechanical Breathing of the Porous Metal Organic Framework MIL-53(Cr): A Computational Exploration Supported by Experiments. J. Phys. Chem. C 2012, 116, 13289-13295.

(38) Coudert, F.; Jeffroy, M.; Fuchs, A. H.; Boutin, A.; MellotDraznieks, C. Thermodynamics of Guest-Induced Structural Transitions in Hybrid Organic-Inorganic Frameworks. J. Am. Chem. Soc. 2008, 130, 14294-14302.

(39) Neimark, A. V.; Coudert, F.; Boutin, A.; Fuchs, A. H. StressBased Model for the Breathing of Metal-Organic Frameworks. J. Phys. Chem. Lett. 2010, 1, 445-449.

(40) Triguero, C.; Coudert, F. X.; Boutin, A.; Fuchs, A. H.; Neimark, A. V. Mechanism of Breathing Transitions in Metal-Organic Frameworks. J. Phys. Chem. Lett. 2011, 2, 2033-2037.

(41) Fairen-Jimenez, D.; Moggach, M. T.; Wharmby, S. A.; Wright, P. A.; Parsons, S.; Düren, T. Opening the Gate: Framework Flexibility in ZIF-8 Explored by Experiments and Simulations. J. Am. Chem. Soc. 2011, 133, 8900-8902.

(42) Grosch, J. S.; Paesani, F. Molecular-Level Characterization of the Breathing Behavior of the Jungle-Gym-Type DMOF-1 MetalOrganic Framework. J. Am. Chem. Soc. 2012, 134, 4207-4215.

(43) Bousquet, D.; Coudert, F. X.; Boutin, A. Free Energy Landscape for the Thermodynamic Understanding of Adsorption-Induced Deformations and Structural Transitions in Porous Materials. J. Chem. Phys. 2012, 137, 044118.

(44) Coudert, F. X.; Mellot-Draznieks, C.; Fuchs, A. H.; Boutin, A. Prediction of Breathing and Gate-Opening Transitions upon Binary Mixture Adsorption in Metal-Organic Frameworks. J. Am. Chem. Soc. 2009, 131, 11329-11332.

(45) Sugiyama, H.; Watanabe, S.; Tanaka, H.; Miyahara, M. Adsorption-Induced Structural Transition of an Interpenetrated Porous Coordination Polymer: Detailed Exploration of Free Energy Profiles. Langmuir 2012, 28, 5093-5100.

(46) Brochard, L.; Vandamme, M.; Pellenq, R. Poromechanics of Microporous Media. J. Mech. Phys. Solids 2012, 60, 606-622.

(47) Boutin, A.; Springuel-Huet, M.; Nossov, A.; Gedeon, A.; Loiseau, T.; Volkringer, C.; Férey, G.; Coudert, F.; Fuchs, A. H. Breathing Transitions in MIL-53(Al) Metal-Organic Framework upon Xenon Adsorption. Angew. Chem., Int. Ed. 2009, 48, 8314-8317.

(48) Coudert, F. X.; Mellot-Draznieks, C.; Fuchs, A. H.; Boutin, A. Double Structural Transition in Hybrid Material MIL-53 upon Hydrocarbon Adsorption: the Thermodynamics behind the Scenes. J. Am. Chem. Soc. 2009, 131, 3442-3443.

(49) Zang, J.; Nair, S.; Sholl, D. S. Osmotic Ensemble Methods for Predicting Adsorption-Induced Structural Transitions in Nanoporous Materials using Molecular Simulations. J. Chem. Phys. 2011, 134, 184103.

(50) Bourrelly, S.; Llewellyn, P. L.; Serre, C.; Millange, F.; Loiseau, T.; Férey, G. Different Adsorption Behaviors of Methane and Carbon Dioxide in the Isotypic Nanoporous Metal Terephthalates MIL-53 and MIL-47. J. Am. Chem. Soc. 2005, 127, 13519-13521. 
(51) Hamon, L.; Llewellyn, P. L.; Devic, T.; Ghoufi, A.; Clet, G.; Guillerm, V.; Pirngruber, G. D.; Maurin, G.; Serre, C.; Driver, G.; van Beek, W.; Jolimaître, E.; Vimont, A.; Daturi, M.; Férey, G. Coadsorption and Separation of $\mathrm{CO}_{2}-\mathrm{CH}_{4}$ Mixtures in the Highly Flexible MIL-53(Cr) MOF. J. Am. Chem. Soc. 2009, 131, 1749017499 .

(52) Neimark, A. V.; Coudert, F.-X.; Triguero, C.; Boutin, A.; Fuchs, A. H.; Beurroies, I.; Denoyel, R. Structural Transitions in MIL-53(Cr): View from Outside and Inside. Langmuir 2011, 27, 4734-41.

(53) Ghoufi, A.; Maurin, G.; Férey, G. Physics Behind the GuestAssisted Structural Transitions of a Porous Metal-Organic Framework. Phys. Chem. Lett. 2010, 1, 2810-2815.

(54) Serra-Crespo, P.; Stavitski, E.; Kapteijn, F.; Gascon, J. High Compressibility of a Flexible Metal-Organic Framework. RSC Adv. 2012, 2, 5051-5053.

(55) Ortiz, A. U.; Boutin, A.; Fuchs, A. H.; Coudert, F. Anisotropic Elastic Properties of Flexible Metal-Organic Frameworks: How Soft are Soft Porous Crystals? Phys. Rev. Lett. 2012, 109, 195502.

(56) Smit, B.; Frenkel, D. Understanding Molecular Simulation; Academic Press: San Diego, 2006.

(57) Vlugt, T. J. H.; Garcia-Perez, E.; Dubbeldam, D.; Ban, S.; Calero, S. Computing the Heat of Adsorption using Molecular Simulations: The Effect of Strong Coulombic Interactions. J. Chem. Theory Comput. 2008, 4, 1107-1118.

(58) Llewellyn, P. L.; Bourrelly, S.; Serre, C.; Filinchuk, Y.; Férey, G. How Hydration Drastically improves Adsorption Selectivity for $\mathrm{CO}_{2}$ over $\mathrm{CH}_{4}$ in the Flexible Chromium Terephthalate MIL-53. Angew. Chem., Int. Ed. 2006, 45, 7751-7754.

(59) Watanabe, S.; Sugiyama, H.; Miyahara, M. Molecular Simulation of Condensation Process of Lennard-Jones Fluids Confined in Nanospace with Jungle-Gym Structure. Adsorption 2008, 14, 165-170.

(60) Watanabe, S.; Sugiyama, H.; Adachi, H.; Tanaka, H.; Miyahara, M. Free Energy Analysis for Adsorption-Induced Lattice Transition of Flexible Coordination Framework. J. Chem. Phys. 2009, 130 (130), 164707.

(61) Liu, Y.-Y.; Couck, S.; Vandichel, M.; Grzywa, M.; Leus, K.; Biswas, S.; Volkmer, D.; Gascon, J.; Kapteijn, F.; Denayer, J. F. M.; Waroquier, M.; Van Speybroeck, V.; Van Der Voort, P. New V V ${ }^{\mathrm{IV}}$-Based Metal-Organic Framework Having Framework Flexibility and High $\mathrm{CO}_{2}$ Adsorption Capacity. Inorg. Chem. 2013, 52, 113-120. 